\title{
ON AUTOMORPHISMS OF LIE ALGEBRAS OF CLASSICAL TYPE. II
}

\author{
BY \\ GEORGE B. SELIGMAN
}

In an earlier paper $\left([22]\left({ }^{1}\right)\right.$, hereafter referred to as $\left.(A)\right)$, we have investigated the automorphisms of Lie algebras of classical type, in the sense of [18]. In Theorem 5 of (A), it was concluded that over an algebraically closed field of characteristic different from 2 and 3, the automorphisms of a simple Lie algebra $\&$ of classical type different from $A_{n}(n \geqq 2), D_{n}(n \geqq 4), E_{6}$, are all invariant automorphisms, that is, are in the group of automorphisms generated by all automorphisms of the form $\exp \left(\operatorname{ad} e_{\alpha}\right)$, where $e_{\alpha}$ runs through all root vectors relative to all standard Cartan subalgebras of $\mathbb{R}$. (For definitions not explained here, see (A) unless otherwise noted.) The results for the remaining simple algebras of classical type were stated in Theorem 6 of (A) without proofs being given. We supply here a proof of this theorem for all cases except that of $E_{6}$, which we shall treat in another paper. In addition, we shall study more closely the automorphisms of Lie algebras of classical type in terms of what appear to be linear representations of minimal dimension.

In the latter connection, the group $\Im(R)$ of invariant automorphisms may be identified in the cases $A-D$ and for sufficiently large characteristics with the quotient, modulo the scalars it contains, of a certain linear group $\mathfrak{E}$, an invariant subgroup in a classical linear group $\mathscr{B}$ such that $\mathfrak{B} / \mathbb{Z}$ is simple, where $\mathbb{B}$ is the center of $\mathfrak{B}$ and consists of scalar transformations. From this identification the isomorphism of $\Im(\mathfrak{R})$ with $\mathfrak{B} / \mathfrak{Z}$ would then follow by the simplicity of $\mathfrak{B} / \mathbb{Z}$; however, another proof of this isomorphism has been chosen, using a proof that $\mathfrak{B}$ is generated by a certain set of elements in the group $\mathfrak{S}$, hence that $\mathfrak{B}=\mathfrak{C}$. The cosets modulo the center of these generators may be identified with the generators for certain of the simple groups $G^{\prime}$ of Chevalley [3] as follows: If $\mathfrak{R}$ is a simple Lie algebra over the field $\mathfrak{F}$ of classical type other than $A_{n}$ with $p \mid(n+1)$, where $p$ is the characteristic of $\mathfrak{F}$, we may obtain $\&$ from the complex simple Lie algebra $\Omega^{*}$ of the same type by taking a basis for $\Omega^{*}$ according to Chevalley, i.e., such that the structure constants are integers. Then we consider the Lie subring $\ell_{1}$ over the integers (over the rationals if $\mathfrak{F}$ is of characteristic zero) generated by this basis, and if $\mathfrak{F}$ is of prime characteristic $p$, we obtain a Lie algebra $\mathfrak{l}_{0}$ over the prime field $Z_{p}$ by reducing coefficients modulo $p$. In every case, we then have a Lie algebra of classical type over the prime field contained in $\mathfrak{F}$, the type being exactly that of $\&$ (see

Received by the editors March 27, 1959.

( ${ }^{1}$ Numbers in brackets refer to the bibliography at the end of the paper. 
$[20 ; 21])$. By extending the base field to $\mathfrak{F}$, we obtain a Lie algebra over $\mathfrak{F}$ isomorphic to $\mathfrak{R}$. If $e_{\alpha}(\alpha \neq 0)$ is a root vector in Chevalley's basis for $\mathfrak{R}^{*}$, the mapping $\exp \left(\lambda \operatorname{ad}\left(e_{\alpha}\right)\right)$ of $\ell^{*}$ maps each vector in the chosen basis into a linear combination of the basis vectors, whereby every coefficient is a polynomial in $\lambda$ with integral coefficients. Reducing these coefficients modulo $p$ if necessary, we obtain polynomials with coefficients in the prime field of $\mathfrak{F}$ which give the coefficients of the images $\left(\bar{h}_{i} ; \bar{e}_{\beta}\right)$ in $\mathfrak{R}$ of the basis $\left(h_{i} ; e_{\beta}\right)$ for $\mathfrak{l}_{1}$ under the mapping $\exp \left(\kappa \operatorname{ad}\left(\bar{e}_{\alpha}\right)\right)$ of $\mathfrak{R}$ into $\mathfrak{R}$, where $\kappa \in \mathfrak{F}$. That is, if the reduction of coefficients in polynomials over the integers $Z$ sends $p(X)$ into $p(X) \in Z_{p}[X]$, and if $u \exp \left(\lambda\right.$ ad $\left.\left(e_{\alpha}\right)\right)=\sum_{i} p_{\alpha, u, i}(\lambda) h_{i}+\sum_{\beta} p_{\alpha, u, \beta}(\lambda) e_{\beta}$ for all vectors $u$ of the above basis for $\mathfrak{l}^{*}$ and for all complex $\lambda$, we have $\bar{u} \exp \left(\kappa \operatorname{ad}\left(\bar{e}_{\alpha}\right)\right)$ $=\sum_{i} \bar{p}_{\alpha, u, i}(\kappa) \bar{h}_{i}+\sum_{\beta} \bar{p}_{\alpha, u, \beta}(\kappa) \bar{e}_{\beta}$ for all $\bar{u}$ and for all $\kappa \in \mathfrak{F}$. The group $G^{\prime}$ of Chevalley is that group of linear transformations of $\&$ generated by the mappings $\bar{u} \rightarrow \sum_{i} \bar{p}_{\alpha, u, i}(\kappa) \bar{h}_{i}+\sum_{\beta} \bar{p}_{\alpha, u, \beta}(\kappa) \bar{e}_{\beta}$ for all $\alpha$, all $\kappa$, hence is the group generated by all mappings $\bar{u} \rightarrow \bar{u} \exp \left(\kappa \operatorname{ad}\left(\bar{e}_{\alpha}\right)\right)$ of $\&$. These mappings define invariant automorphisms of $\mathfrak{R}$, so that $G^{\prime}$ may be identified with a subgroup of $\Im(\mathfrak{R})$. In the cases where $\mathfrak{R}$ is of types $A-D$, Ree [19] has used results of Dickson $[6 ; 7]$ on the generation of certain classical groups to show that a certain group $\mathfrak{S}$ of linear transformations, which modulo its scalars is isomorphic to $G^{\prime}$, is a classical linear group, which in at least some cases is the group $\mathfrak{B}$ which we encounter. In the case of type $G$, he has also given such a realization for a group $\mathfrak{S}$ as above. In the cases of types $B, D$ and $G$, his realizations of $\mathfrak{R}^{*}$ lead to an interpretation of the group we have denoted by $\mathfrak{S}$ as a group of linear transformations in a space at least formally different from that where our group $\mathfrak{B}$ acts, and in the case $p \mid(n+1)$ of $A_{n}$, we cannot directly regard $G^{\prime}$ as a subgroup of $\Im(\mathfrak{R})$. In this paper, these difficulties are overcome, and we show that the relation $G^{\prime} \cong \mathfrak{I}(\mathfrak{R}) \cong \mathfrak{B} / \mathbb{Z}$ holds without exception in the cases $A, B, C, D, G$. (Hence, since $G^{\prime}$ is simple [3], we have another proof that $\mathfrak{B} / \mathbb{B}$ is.) Analogous results for some of the remaining exceptional algebras will appear in another paper.

Note. The referee has observed that in the algebraically closed case the result that $G^{\prime}=\Im(R)$ can be deduced from the theory of algebraic groups, as can the result $\mathfrak{Y}(\mathfrak{R})=\mathfrak{B} / \mathfrak{Z}$ in some cases (Ono, Takashi, Sur les groupes de Chevalley, J. Math. Soc. Japan vol. 10 (1958) p. 307). However, the effectiveness of this method is lost when arbitrary fields are considered.

In the cases of types $A_{n}(p \nmid(n+1)), B, C, D_{n}(n \geqq 5)$, the full automorphism group $\mathfrak{A}(\mathfrak{Q})$ of $\mathfrak{Z}$ has been determined by Jacobson $[12 ; 14]$, as well as for type $G$ if $\mathfrak{F}$ is of characteristic zero [13]; for type $F$ and characteristic zero, Tomber [24] has made this determination. In these papers, the principal tool has been the demonstration that the algebra in question has only one or two inequivalent irreducible representations of the degree of the representation used to define the algebra. The techniques involve counting weights and are particularly unwieldy in the case of prime characteristic. Here, at the 
sacrifice of some low characteristics, the representation-theoretic approach is eliminated by replacing it with the results of (A) and a device of $\$ 1$ below. Using this new technique, we have complete results on the full automorphism group for Lie algebras of classical types $A-D, G_{2}, F_{4}$ and $E_{6}$, although the latter two are not discussed in this paper. For the first five types, we also describe here the quotient group $\mathfrak{A}(\mathfrak{R}) / \mathfrak{Y}(\mathfrak{l})$.

1. Preliminaries. Let $\mathfrak{F}$ be a field of characteristic different from $2, \mathfrak{B}$ a vector space over $\mathfrak{F}$. Let $\mathfrak{F}(\mathfrak{B})$ be the set of all $\mathfrak{F}$-linear transformations of $\mathfrak{B}$, and let $T \in \mathbb{E}(\mathfrak{B})$. Let $\mathfrak{S}$ be a subspace of $\mathbb{E}(\mathfrak{B})$ such that $U \operatorname{ad}(T)=[U T]$ $=U T-T U \in \mathfrak{S}$ for all $U \in \mathfrak{S}$. Suppose further that either: (i) $\mathfrak{F}$ is of characteristic 0 and $T^{k}=0$ for some integer $k\left(\right.$ then $\left.(\operatorname{ad}(T))^{2 k-1}=0\right)$, or (ii) $\mathfrak{F}$ is of odd prime characteristic $p$ and $T^{(p+1) / 2}=0\left(\operatorname{then}(\operatorname{ad}(T))^{p}=0\right)$. Then in either case both $\exp (T)$ and $\exp (\operatorname{ad}(T))$ are defined, the former acting in $\mathfrak{B}$ and the latter in $\mathfrak{S}$, by the power series $\exp (A)=I+\sum_{i=1}^{\infty}(i !)^{-1} A^{i}$, where $(i !)^{-1} A^{i}$ is defined to be zero whenever $A^{i}=0$, regardless of whether $(i !)^{-1}$ is defined. If $T$ is as in (i) or (ii), then $\exp (T)$ is of the form $I+N$, where $N$ is nilpotent; hence $\exp (T)$ is nonsingular, and if $\mathfrak{B}$ is finite-dimensional, then $\operatorname{det}(\exp (T))=1$. The same observations of course hold when $T$ is replaced by $\operatorname{ad}(T)$, considered as a linear transformation of $\mathfrak{S}$. Subject to the assumption that $T$ is as in (i) or (ii), the following formulas are readily derived:

(1) $(\exp T)^{-1}=\exp (-T)$;

(2) If $U \in \subseteq$, then $U \exp (\operatorname{ad} T)=(\exp T)^{-1} U(\exp T)$;

(3) If $U, V$ and $U V$ are in $\mathfrak{\subseteq}$, then $(U V) \exp (\operatorname{ad} T)=(U \exp (\operatorname{ad} T))$ $\cdot(V \exp (\operatorname{ad} T))$;

(4) If $U, V$ and $[U V]$ are in $\mathfrak{S}$, then

$$
[U V] \exp (\operatorname{ad} T)=[U \exp (\operatorname{ad} T), V \exp (\operatorname{ad} T)] .
$$

The formula (1) is obtained by observing that the coefficients of all powers of $T$ in the expanded form of $(\exp T)(\exp (-T))$ are zero, with the exception of that of $T^{0}=I$, which is 1 . The formula (2) is obtained by collecting and comparing the terms of the same total degree in $T$ in the two series obtained when $\exp (-T)$ is substituted for $(\exp T)^{-1}$ on the right-hand side, and when the left-hand side is expanded using the rule

$$
U(\operatorname{ad} T)^{n}=\sum_{i=0}^{n}(-1)^{i}\left(\begin{array}{c}
n \\
i
\end{array}\right) T^{i} U T^{n-i} .
$$

Then (3) and (4) follow at once from (2).

If $\mathfrak{B}$ is an algebra (not necessarily associative) over $\mathfrak{F}$, if $\mathfrak{S}$ is the Lie algebra of all derivations of $\mathfrak{B}$ over $\mathfrak{F}$, and if $T \in \mathfrak{S}$ satisfies either condition (i) or (ii), then for all $x, y \in \mathfrak{B}$, we have (the product in $\mathfrak{B}$ being $x y$ ):

$$
(x y) T^{n}=\sum_{k=0}^{n}\left(\begin{array}{l}
n \\
k
\end{array}\right)\left(x T^{k}\right)\left(y T^{n-k}\right),
$$


and from this we derive the formula

$$
(x y) \exp (T)=(x \exp (T))(y \exp (T))
$$

by showing that both sides are equal to

$$
\sum_{n=0}^{p-1} \sum_{i+j=n} i !^{-1} j !^{-1}\left(x T^{i}\right)\left(y T^{j}\right)
$$

in the case (ii). The result is classical in the case (i), and is proved by the same method. Since $\exp (T)$ is nonsingular, we see that it is an automorphism of $\mathfrak{B}$.

If $\mathfrak{B}$ again is simply a vector space over $\mathfrak{F}$, and if we have an $m$-linear function on $\mathfrak{B}$ over $\mathfrak{F}$ with values in a vector space $\mathfrak{B}$ over $\mathfrak{F}$, i.e., a function $\left(x_{1}, \cdots, x_{m}\right)$ of $m$ variables, linear in each variable separately, we let $\varsigma$ be the set of linear transformations $U$ of $\mathfrak{B}$ satisfying

$$
\left(x_{1} U, x_{2}, \cdots, x_{m}\right)+\cdots+\left(x_{1}, \cdots, x_{m-1}, x_{m} U\right)=0
$$

for all $x_{1}, \cdots, x_{m} \in \mathfrak{B}$. If $U$ and $V$ are in $\subseteq$, then we may apply (6) to show that $[U V] \in \mathfrak{S}$, so that $\mathfrak{S}$ is a Lie algebra of linear transformations of $\mathfrak{B}$. Now if $T \in S$ satisfies (i) or (ii) ${ }^{\prime} T^{r+1}=0$ for some $r<p / m$, and if $A=\exp (T)$, we have the formula

$$
\left(x_{1} A, x_{2} A, \cdots, x_{m} A\right)=\left(x_{1}, x_{2}, \cdots, x_{m}\right)
$$

for all $x_{1}, x_{2}, \cdots, x_{m} \in \mathfrak{B}$. We outline the proof in the case (ii)'. Substituting $\exp (T)$ for each $A$ on the left-hand side and collecting all terms of total degree $n$ in $T$, we find that this homogeneous term is

$$
\sum_{i_{1}+\cdots+i_{m}=n}\left(i_{1} !^{-1} \cdots i_{m} !-1\right)\left(x_{1} T^{i_{1}}, \cdots, x_{m} T^{i_{m}}\right),
$$

where $0 \leqq i_{j} \leqq r$ for each $j, 1 \leqq j \leqq m$; therefore we may assume $n \leqq m r<p$. For $n=0$, this is $\left(x_{1}, \cdots, x_{m}\right)$. Thus we need only show that (8) vanishes for all $n>0, n<p$; furthermore, instead of restricting $i_{m}$ to the range $0 \leqq i_{m} \leqq r$, we can allow it to run over the range $0 \leqq i_{m} \leqq n$; for $n<p$ assures us that $i_{m} !^{-1}$ is defined, and $T^{r+1}=0$ assures that all the new terms obtained in (8) are zero. Next one proves by induction on $j$ that if $0 \leqq k \leqq p$, if $0 \leqq j \leqq k$, and if $u_{1}, \cdots$, $u_{m} \in \mathfrak{B}$, then

$$
\begin{aligned}
& \left(u_{1}, \cdots, u_{m-1}, u_{m} T^{k}\right) \\
& \quad=(-1)^{i} \sum_{n_{1}+\cdots+v_{m-1}=j}\left(j ! \nu_{1} !^{-1} \cdots \nu_{m-1} !^{-1}\right)\left(u_{1} T^{\nu_{1}}, \cdots, u_{m-1} T^{\nu_{m-1}}, u_{m} T^{k-j}\right)
\end{aligned}
$$

(with the convention that $p ! p !^{-1}=1$ ), which for $k=j$ is

$$
\begin{aligned}
\left(u_{1}, \cdots, u_{m-1}, u_{m} T^{k}\right) & \\
& =(-1)^{k} \sum_{v_{1}+\cdots+v_{m-1}=k}\left(k ! v_{1} !^{-1} \cdots \nu_{m-1} !^{-1}\right)\left(u_{1} T^{\nu 1}, \cdots, u_{m-1} T^{\nu_{m}-1}, u_{m}\right) .
\end{aligned}
$$


If we now apply this result by substitution in (8), we see that (8) is equal to

$$
\begin{aligned}
& \sum_{i_{1}+\cdots+i_{m}=n} \sum_{\nu_{1}+\cdots+v_{m-1}=i_{m}}(-1)^{i_{m}}\left(i_{1} !^{-1} \nu_{1} !^{-1} \cdots i_{m-1} !^{-1} \nu_{m-1} !^{-1}\right) \\
&\left(x_{1} T^{i_{1}+\nu_{1}}, \cdots, x_{m-1} T^{i_{m-1}+\nu_{m-1}}, x_{m}\right),
\end{aligned}
$$

where $i_{j} \leqq r, 1 \leqq j \leqq m-1, i_{m} \leqq n$. Setting $k_{j}=i_{j}+\nu_{j}, 1 \leqq j \leqq m-1$, and noting that any terms of (11) with some $k_{j}>r$ are zero, we compute the coefficient of $\left(x_{1} T^{k_{1}}, \cdots, x_{m-1} T^{k_{m-1}}, x_{m}\right)$ in (11) for each fixed $(m-1)$-tuple $\left(k_{1}, \cdots, k_{m-1}\right)$ with $0 \leqq k_{j} \leqq r$ for all $j$. This coefficient is

$$
\begin{aligned}
\sum_{i_{1}+\cdots+i_{m}=n}(-1)^{\left(k_{1}-i_{1}\right)+\cdots+\left(k_{m-1}-i_{m-1}\right)} i_{1} !^{-1}\left(k_{1}-i_{1}\right) !^{-1} \cdots & \\
& i_{m-1} !^{-1}\left(k_{m-1}-i_{m-1}\right) !^{-1},
\end{aligned}
$$

where $0 \leqq i_{j} \leqq k_{j}, 1 \leqq j \leqq m-1$, and where $0 \leqq i_{m} \leqq n$. But since $i_{m}$ runs over the entire range from 0 to $n$, and since $k_{1}+\cdots+k_{m-1}=n$, every $(m-1)$-tuple $\left(i_{1}, \cdots, i_{m-1}\right)$ with $0 \leqq i_{j} \leqq k_{j}, 1 \leqq j \leqq m-1$, occurs in this sum. Thus the sum (12) is

$$
\begin{aligned}
\sum_{i_{1}=0}^{k_{1}} \ldots & \sum_{i_{m-1}=0}^{k_{m-1}}(-1)^{k_{1}-i}\left(i_{1} !^{-1}\left(k_{1}-i_{1}\right) !^{-1}\right) \cdots(-1)^{k_{m-1}-i_{m-1}} \\
& \cdot\left(i_{m-1}{ }^{-1}\left(k_{m-1}-i_{m-1}\right) !^{-1}=\prod_{j=1}^{m-1} \sum_{i_{j}=0}^{k_{j}}(-1)^{k_{j}-i_{j}}\left(i_{j} !^{-1}\left(k_{j}-i_{j}\right) !^{-1}\right) .\right.
\end{aligned}
$$

Now $\sum k_{j}=n>0$, so some $k_{j}>0, k_{j}<p$, and thus

$$
\sum_{i_{j}=0}^{k_{j}}(-1)^{k_{j}-i_{j}}\left(i_{j} !^{-1}\left(k_{j}-i_{j}\right) !^{-1}\right)=0
$$

for this $j$. Therefore the product is zero, and the proof is complete.

Finally we note a device which we shall use to establish the vanishing of a low power of a nilpotent linear transformation $T$.

Lemma 1. Let $\mathfrak{S}$ be a Lie subalgebra of $\mathbb{E}(\mathfrak{B})$ such that for some $k, \mathfrak{F} I+\mathfrak{S}$ $+\mathfrak{S}^{2}+\cdots+\mathfrak{S}^{k}=\mathfrak{R}$ is an irreducible algebra of linear transformations in $\mathfrak{B}$, where the exponents denote associative powers. Let $T$ be a nilpotent linear transformation of $\mathfrak{B}$ lying in $\mathfrak{S}$. Suppose that $(\operatorname{ad} T)^{j}=0$, acting in $\mathfrak{S}$. Then $T^{k(j-1)+1}=0$.

Proof. First we observe that if $\mathfrak{B}_{i}$ is the null-space of $T^{\boldsymbol{i}}$ in $\mathfrak{B}$, then $\mathfrak{B}_{i} \Im^{m} \subseteq \mathfrak{B}_{i+m(j-1)}$ for all $m \geqq 1$. It evidently suffices to prove the assertion for $m=1$. Therefore let $x \in \mathfrak{B}_{i}, U \in \mathfrak{S}$; then one readily proves by induction on $n$ that

$$
x U T^{n}=\sum_{\nu=0}^{n}\left(\begin{array}{l}
n \\
\nu
\end{array}\right) x T^{\nu}\left(U(\operatorname{ad} T)^{n-\nu}\right) .
$$


For $n=i+j-1$, those terms with $\nu \geqq i$ are zero since $x \in \mathfrak{B}_{i}$; those with $\nu<i$, i.e., with $i+j-1-\nu \geqq j$, are zero since $(\operatorname{ad} T)^{j}=0$. Thus $x U T^{i+j-1}=0$, and $\mathfrak{B}_{i} \subseteq \subseteq \mathfrak{B}_{i+j-1}$; this is our assertion for $m=1$.

Now evidently $\mathfrak{B}_{i}(\mathfrak{F} I)=\mathfrak{B}_{i} \subseteq \mathfrak{B}_{i+r}$ for all $r>0$. Thus $\mathfrak{B}_{1} \mathfrak{R} \subseteq \mathfrak{B}_{1+k(j-1)}$. But $\mathfrak{B}_{1} \Re$ is an $\Re$-invariant subspace of $\mathfrak{B}$, hence is either 0 or $\mathfrak{B}$, and $\mathfrak{B}_{1} \neq 0$ since $T$ is nilpotent. Since $I \in \Re, \mathfrak{B}_{1} \Re=\mathfrak{B} \subseteq \mathfrak{B}_{k(j-1)+1}$, or $T^{k(j-1)+1}=0$ on $\mathfrak{B}$, as asserted.

2. Algebras of type $A$. If the characteristic of $\mathfrak{F}$ is not 2,3 or a prime dividing $n$, and if $n>1$, let $\&$ be the Lie algebra (under $A B-B A$ ) of all $n$ by $n \mathfrak{F}$-matrices of trace zero. Then $\mathfrak{R}$ is a simple Lie algebra of classical type $A_{n-1}$ (see, e.g., $\left.[11 ; 14 ; 20]\right)$. A particular Cartan decomposition relative to a standard Cartan subalgebra $\mathfrak{S}$ arises when we take $\mathfrak{S}$ to be the set of diagonal matrices in $\&$; then there are $n^{2}-n$ distinct roots, each root-space being spanned by a matrix unit $E_{i j}, i \neq j$. If the characteristic $p$ of $\mathfrak{F}$ is a prime greater than 3 and dividing $n$, let $\&$ be the quotient of the Lie algebra of $n$ by $n \mathfrak{F}$-matrices of trace zero by the ideal consisting of all scalar multiples of the identity matrix. Then $\mathfrak{R}$ is simple of classical type $A_{n-1}[14 ; 21]$, with a particular standard Cartan decomposition being obtained by taking the Cartan subalgebra $\mathfrak{S}$ to be the cosets modulo $\mathfrak{F} I$ of the diagonal matrices of trace zero, and with the cosets of the matrix units $E_{i j}, i \neq j$, belonging to the $n^{2}-n$ distinct roots. It follows by the isomorphism theorem (Theorem 9 ) of [18] that these exhaust all simple Lie algebras of classical type $A$ over $\mathfrak{F}$.

First let $\mathfrak{F}$ be algebraically closed. If $n=2$, we know by Theorem 5 of (A) that the automorphism group $\mathfrak{A}$ of $\mathbb{R}$ coincides with the group $\mathfrak{Y}$ generated by all $\exp \left(\operatorname{ad} E_{\alpha}\right)$, where $E_{\alpha}$ runs through all root-vectors belonging to nonzero roots relative to standard Cartan subalgebras (the group of invariant automorphisms). If $n>2$, we know from (A) that the index of $\Im$ in $\mathfrak{A}$ is 1 or 2 . Let us temporarily exclude the case where the characteristic $p$ of $\mathfrak{F}$ divides $n$. Then $\mathbb{R}$ can be viewed as the Lie algebra of all linear transformations of trace zero in an $n$-dimensional vector space $\mathfrak{B}$, and we have $\mathfrak{F} I+\mathfrak{l}=\mathfrak{E}(\mathfrak{B})$. Moreover, $\left(\operatorname{ad} E_{\alpha}\right)^{3}=0$ for all $E_{\alpha}$ of the above type, and $\mathbb{R}$ is a restricted Lie algebra (under $p$ th iterates) in the sense of Jacobson $[10 ; 14]$ if the characteristic of $\mathfrak{F}$ is a prime $p$. Since $\left(\operatorname{ad} E_{\alpha}\right)^{p}=0=\operatorname{ad}\left(E_{\alpha}^{p}\right)$, we have $E_{\alpha}^{p}=0$ in this case; in the case of characteristic zero, the fact that $E_{\alpha}$ is nilpotent follows by the classical representation theory of semi-simple Lie algebras $[2 ; 9 ; 23 ; 26]$. Thus we can apply Lemma 1 to conclude that $E_{\alpha}^{1(3-1)+1}=E_{\alpha}^{3}=0$. Therefore all formulas of $\S 1$ which involved the hypothesis $T^{2^{-1}(p+1)}=0$ are valid for $T=E_{\alpha}$ whenever $p \neq 2,3$ (and, of course, all formulas are valid in the case of characteristic zero). We therefore have, by (2), $U \exp \left(\operatorname{ad} E_{\alpha}\right)=\left(\exp E_{\alpha}\right)^{-1} U\left(\exp E_{\alpha}\right)$ for all $U \in \mathbb{R}$, where $\exp E_{\alpha}$ is unimodular. If $A \in \mathbb{E}(\mathfrak{B})$ is nonsingular, then the mapping $U \rightarrow A^{-1} U A$ is an automorphism of $\mathfrak{R}$, and we have $A^{-1} U A=U$ for all $U \in \mathbb{R}$ if and only if $A$ is scalar, by the fact that $\mathfrak{F} I+\mathbb{R}=\mathfrak{E}(\mathfrak{B})$. By taking $B$ to be a suitable scalar multiple of $A$, we have $B \in S L(\mathfrak{B})$, the unimodular 
group, and $A^{-1} U A=B^{-1} U B$ for all $U \in R$. Via this correspondence, we see that the automorphism group $\mathfrak{R}$ of $\mathbb{R}$ contains as a subgroup the projective unimodular group $P S L(\mathfrak{B})$. All mappings $\exp \left(\operatorname{ad} E_{\alpha}\right)$ are in this subgroup; hence we have $\Im \subseteq P S L(\mathfrak{B}) \subseteq \mathfrak{A}$.

If $U^{\prime}$ denotes the ordinary transpose of the matrix $U$, the mapping $U \rightarrow-U^{\prime}$ is also an automorphism of $\mathbb{R}$. If $n=2$, we have $A^{-1} U A=-\dot{U}^{\prime}$ for all $U \in \mathbb{R}$, where

$$
A=\left(\begin{array}{rr}
0 & 1 \\
-1 & 0
\end{array}\right)
$$

and since $\mathfrak{F}=\mathfrak{R}$, the full automorphism group of $\mathfrak{R}$ is $P S L(\mathfrak{B})$. If $n>2$, suppose there is a nonsingular $A \in \mathbb{E}(\mathfrak{B})$ such that $A^{-1} U A=-U^{\prime}$ for all $U \in \mathbb{R}$. Then $-E_{31}=A^{-1} E_{13} A=A^{-1} E_{12} E_{23} A=A^{-1} E_{12} A A^{-1} E_{23} A=E_{21} E_{32}=0$, which is absurd. Hence the automorphism $U \rightarrow-U^{\prime}$ is not in $P S L(\mathfrak{B})$, therefore not in $\Im$, the index of $\Im$ in $\mathfrak{A}$ is $2, \mathfrak{Y}=P S L(\mathfrak{B})$, and the full group $\mathfrak{A}$ may be regarded as the product (not direct) of the group of order 2 generated by $U \rightarrow-U^{\prime}$ by the invariant subgroup $P S L(\mathfrak{B})$.

Still excluding the case $p \mid n$, let us now drop the assumption that $\mathfrak{F}$ is algebraically closed, and let $\Omega$ be its algebraic closure. Then $\ell_{\Omega}$, the algebra over $\Omega$ obtained by extending the base field, is the set of $\Omega$-linear transformation of trace zero in $\mathcal{E}\left(\mathfrak{B}_{\Omega}\right)$. If $\sigma$ is an automorphism of $\mathcal{R}$, then $\sigma$ is defined on a basis for $\mathfrak{l}_{\Omega}$ whose corresponding structure constants are in $\mathfrak{F}$, and determines a unique extension to an automorphism of $\mathfrak{R}_{\Omega}$, again denoted by $\sigma$. By our previous results, we have either $U^{\sigma}=A^{-1} U A$ for all $U \in \mathbb{R}_{\Omega}$ or $U^{\sigma}$ $=-A^{-1} U^{\prime} A$ for all $U \in \mathfrak{R}_{\Omega}$, where $A$ is some nonsingular element of $\left(\&\left(\mathfrak{B}_{\Omega}\right)\right.$. Thus for all $U \in \mathbb{R}$, we have either $U^{\sigma}=A^{-1} U A \in \mathbb{R}$ or $-U^{\sigma}=A^{-1} U^{\prime} A \in \mathbb{R}$ for some $A \in \mathcal{E}\left(\mathfrak{B}_{\Omega}\right)$. It follows by a well-known result $[25$, p. 70$]$ that either there is a nonsingular $B \in \mathbb{E}(\mathfrak{B})$ such that $U^{\sigma}=B^{-1} U B$ for all $U \in \mathbb{R}$ or there is a $B \in \mathbb{E}(\mathfrak{B})$ such that $U^{\sigma}=-B^{-1} U^{\prime} B$ for all $U \in \mathbb{R}$, with the former being the case if $n=2$. Since $\mathfrak{F} I+\mathbb{R}=\mathfrak{E}(\mathfrak{B})$ still holds, we see that the automorphism group $\mathfrak{A}$ of $\mathfrak{R}$ is isomorphic in the above fashion to the projective linear group $P L(\mathfrak{B})$ if $n=2$, and to the product of $P L(\mathfrak{B})$ by the group of order 2 generated by $U \rightarrow-U^{\prime}$ if $n>2$. The invariant automorphisms form a normal subgroup of $\mathfrak{A}$ contained in $P S L(\mathfrak{B})$ as before. Thus $\mathfrak{Y}=P S L(\mathfrak{B})$ will follow directly from the simplicity of $P S L(\mathfrak{B})$; however, we prefer in the following paragraphs to apply the reasoning of Chevalley [3] and of Ree [19].

If $\mathfrak{S}_{0}$ is a second standard Cartan subalgebra of $\mathfrak{R}$, then it follows by the absence of isomorphisms among the distinct classical types $[12 ; 14 ; 21]$ that $\mathfrak{S}_{0}$ has the same dimension as does $\mathfrak{S}$, the diagonal subalgebra, and a fundamental system of roots of the same type. Hence by Theorem 9 of [18] there is an automorphism of $\mathfrak{R}$ mapping $\mathfrak{S}$ onto $\mathfrak{S}_{0}$; since $-X^{\prime} \in \mathfrak{S}$ for all $X \in \mathfrak{S}$, there is an automorphism of the form $U \rightarrow A^{-1} U A$ of $\mathfrak{R}$ mapping $\mathfrak{S}$ onto $\mathfrak{S}_{0}$.

In this case, the simple group $G^{\prime}$ of Chevalley [3] is that generated by the 
$\exp \left(\operatorname{ad} E_{\alpha}\right)$, where $E_{\alpha}$ runs through all root-vectors belonging to nonzero roots $\alpha$ relative to a fixed standard Cartan subalgebra $\mathfrak{S}_{0}$ of $\mathfrak{R}$. (For the passage from complex Lie algebras to arbitrary fields, see also the papers of Curtis $[4 ; 5]$ and of Ree [19].) Let $e_{1}, \cdots, e_{n}$ be a basis for $\mathfrak{B}, \mathfrak{S}$ the diagonal Cartan subalgebra of $\mathfrak{l}$ relative to this basis, and let $A \in \mathbb{E}(\mathfrak{B})$ have $\mathfrak{S}_{0}$ $=A^{-1} \mathfrak{S} A$ as before. Then $\mathfrak{S}_{0}$ is the diagonal Cartan subalgebra relative to the basis $e_{1} A, \cdots, e_{n} A$, and the $E_{\alpha}$ are the scalar multiples $\lambda E_{i j}$ of the matrix units $E_{i j}$ relative to this basis. Thus $\exp \left(E_{\alpha}\right)=\exp \left(\lambda E_{i j}\right)=I+\lambda E_{i j}, i \neq j$. It is well known that these transformations generate the full group $S L(\mathfrak{B})[7 ; 19]$. Thus Chevalley's group $G^{\prime}$ contains all automorphisms of $\mathbb{R}$ of the form $U \rightarrow A^{-1} U A, A \in S L(\mathfrak{B})$, i.e., $G^{\prime} \supseteq P S L(\mathfrak{B}) \supseteq \Im$. But evidently $G^{\prime} \subseteq \Im$, so we have $G^{\prime}=P S L(\mathfrak{B})=\Im$. The first of these equalities was demonstrated by Ree [19].

Now let $\mathfrak{R}$ be as in the case where $\mathfrak{F}$ is of characteristic $p$ with $p \mid n$, and first let $\mathfrak{F}$ be algebraically closed. Then $\mathfrak{R}$ is again a restricted Lie algebra, under taking cosets of ordinary pth powers of matrices of trace zero [14]. Again we have $\left(\operatorname{ad} E_{\alpha}\right)^{3}=0$, hence $E_{\alpha}^{[p]}=0$, where the bracket notation is used to indicate the $p$ th power in $\mathcal{Q}$ defined above. If $X$ is any representative in the algebra $\mathfrak{M}$ of matrices of trace zero of the coset $E_{\alpha} \in \mathfrak{R}$, we thus have $X^{p}=\lambda I, \lambda \in \mathfrak{F}$. Then $X-\lambda^{p^{-1}} I$ also represents $E_{\alpha}$ in $\mathfrak{M}$, and we have $\left(X-\lambda^{p^{-1}} I\right)^{p}=0$. Moreover, we have $\mathfrak{F} I+\mathfrak{M}+\mathfrak{M}^{2}=\mathfrak{E}(\mathfrak{B})$, where $\mathfrak{B}$ is an $n$-dimensional vector space where $\mathfrak{M}$ acts, while from $\left(\operatorname{ad} E_{\alpha}\right)^{3}=0$ in $\mathfrak{R}$, we find that $\left(\operatorname{ad}\left(X-\lambda^{p^{-1}} I\right)\right)^{4}=0$, acting in $\mathfrak{M}$. By Lemma 1, we have $\left(X-\lambda^{p^{-1}} I\right)^{7}$ $=0$. Thus our equations of $\S 1$ involving the hypothesis $T^{2^{-1}(p+1)}=0$ can only be applied to $T=X-\lambda^{p^{-1}} I$ if $p \geqq 13$. We shall assume that this is the case in the sequel. We replace $X-\lambda^{p^{-1}} I$ by the notation $E_{\alpha}^{*}$; thus $E_{\alpha}^{*}$ is a representative of the coset $E_{\alpha}$ with $E_{\alpha}^{* 7}=0$. If $U \in \mathbb{R}$, and if $U^{*}$ is a representative of $U$ in $\mathfrak{M}$, then the mapping

$$
U \rightarrow \phi\left(U^{*} \exp \left(\operatorname{ad} E_{\alpha}^{*}\right)\right)=\phi\left(\exp \left(-E_{\alpha}^{*}\right) U^{*} \exp \left(E_{\alpha}^{*}\right)\right),
$$

where $\phi$ is the canonical homomorphism of $\mathfrak{M}$ onto $\mathfrak{R}$, is an automorphism of $\mathfrak{R}$ which coincides with $\exp \left(\operatorname{ad} E_{\alpha}\right)$, and $\exp \left(E_{\alpha}^{*}\right)$ is unimodular. If we have $U=\phi\left(A^{-1} U^{*} A\right)$ for all $U \in \mathbb{R}$, we have $U^{*}=A^{-1} U^{*} A+\lambda\left(U^{*}\right) I$ for all $U^{*} \in \mathfrak{M}$, or $A^{-1} U^{*} A=U^{*}-\lambda\left(U^{*}\right) I$, where $\lambda\left(U^{*}\right) \in \mathfrak{F}$. For $U^{*}=E_{i j}, i \neq j$, we have $\left(A^{-1} E_{i j} A\right)^{2}=A^{-1} E_{t j}^{2} A=0$, while $E_{i j}-\lambda\left(E_{i j}\right) I$ is nonsingular unless $\lambda\left(E_{i j}\right)=0$. Thus $A^{-1} E_{i j} A=E_{i j}$ for all $i \neq j$. It follows that $A$ is in the center of $\mathscr{E}(\mathfrak{B})$, i.e., $A \in \mathfrak{F} I$. Thus the group $\mathfrak{Y}$ of invariant automorphisms of $\mathbb{R}$ may be again considered as a subgroup of $P S L(\mathfrak{B})$ via the identification of $\exp \left(\operatorname{ad} E_{\alpha}\right)$ with the coset of $\exp \left(E_{\alpha}^{*}\right)$ in $S L(\mathfrak{B})$ by the subgroup of scalars in $S L(\mathfrak{B})$ as utilized above. The mapping $U \rightarrow-\phi\left(U^{* \prime}\right)$ is likewise an automorphism of $\mathscr{R}$, and if $A^{-1} U^{*} A=\lambda\left(U^{*}\right) I-U^{* \prime}$ for all $U^{*} \in \mathfrak{M}$, we must again have $\lambda\left(E_{i j}\right)=0$ for all $i \neq j, A^{-1} E_{i j} A=-E_{j i}$. As before, this is impossible. Thus the automorphism 
group $\mathfrak{A}$ properly contains $P S L(\mathfrak{B})$, which contains $\Im$; since the index of $\Im$ is at most 2 , we again have $\mathfrak{F}=P S L(\mathfrak{B})$, and $\mathfrak{Y}$ is of index 2 in $\mathfrak{A}$.

If $\mathfrak{F}$ is not assumed to be algebraically closed, and if $\Re$ is its algebraic closure as before, with $\mathfrak{M}$ the Lie algebra of $n$ by $n \mathfrak{F}$-matrices of trace zero, then $\mathfrak{M}_{\Omega}$ is the Lie algebra of $n$ by $n \Omega$-matrices of trace zero, and the map̀ping $X+\mathfrak{F} I \rightarrow X+\Omega I(X \in \mathfrak{M})$ of $\mathfrak{R}$ into $\mathfrak{M}_{\Omega} / \Omega I$ is readily seen to be an isomorphism of Lie algebras over $\mathfrak{F}$. Since $\mathfrak{M}_{\Omega} / \Omega I$ is a Lie algebra over $\Re$, this isomorphism may be uniquely extended to a $\Omega$-isomorphism of $\Omega_{\Omega}$ into $\mathfrak{M}_{\Omega} / \Omega I$; but now both algebras have the dimension $n^{2}-2$ over $\Omega$; hence $\mathfrak{R}_{\Omega}$ is isomorphic to $\mathfrak{M}_{\Omega} / \Omega I$ via the mapping sending an element $\sum \alpha_{t}\left(X_{i}+\mathfrak{F} I\right)$ with $\alpha_{i} \in \Omega, X_{i} \in \mathfrak{M}$, into $\sum \alpha_{i}\left(X_{i}+\Omega I\right)=\left(\sum \alpha_{i} X_{i}\right)+\Omega I$. We shall regard $\mathfrak{R}_{\Omega}$ as identified with $\mathfrak{M}_{\Omega} / \Omega I$ through this mapping. We let $\phi$ be the canonical homomorphism (over $\Re$ ) of $\mathfrak{M}_{\Re}$ on to $\mathfrak{R}_{\Omega}, \psi$ that (over $\mathfrak{F}$ ) of $\mathfrak{M}$ onto $\mathbb{R}$. If $\sigma$ is an automorphism of $\mathcal{R}$, we also denote by $\sigma$ its unique extension to an automorphism of $\mathfrak{l}_{\Omega}$, and we have either $U^{\sigma}=\phi\left(A^{-1} U^{*} A\right)$ or $U^{\sigma}=-\phi\left(A^{-1} U^{* \prime} A\right)$ for all $U \in \Omega_{\Omega}$, where $A$ is nonsingular in the ring $\&\left(\mathfrak{B}_{\Omega}\right)$, and where $U^{*} \in \mathfrak{M}_{\Omega}$ is any element with $\phi\left(U^{*}\right)=U$. In particular, if $U^{*}$ is a matrix unit $E_{i j}$, $i \neq j$, and if $U^{\sigma}=X+\Re I, X \in \mathfrak{M}$ (since the coset of $E_{i j}$ is in $\mathfrak{l}$ ), we have in the first case, $\phi(X)=\phi\left(A^{-1} E_{i j} A\right)$, or $A^{-1} E_{i j} A=X+\lambda I, \lambda \in \Omega$. Since $\left(A^{-1} E_{i j} A\right)^{2}$ $=0$, we have $(X+\lambda I)^{2}=0$. If $X=\left(\xi_{\mu \nu}\right)$ and if some $\xi_{\mu \nu} \neq 0$ for $\mu \neq \nu$, then the entry in the $\mu$ th row, $\nu$ th column of $(X+\lambda I)^{2}$ is $2 \lambda \xi_{\mu \nu}+\sum_{\rho} \xi_{\mu \rho} \xi_{\rho \nu}=0$, and $\lambda \in \mathfrak{F}$. If $X$ is diagonal, then since $-\lambda$ is an eigenvalue of $X$, we have $X+\lambda I$ $=0$, and $\lambda \in \mathfrak{F}$. Thus in either case $A^{-1} E_{i j} A \in \mathfrak{M}$ for all $E_{i j}$ with $i \neq j$, hence $A^{-1} \mathfrak{M} A \subseteq \mathfrak{M}$. The same conclusion is valid when $\sigma$ is of the second form, since $\mathfrak{M}$ is closed under the taking of negative transposes. Therefore there is a nonsingular $B \in \mathfrak{F}(\mathfrak{B})$, the full $\mathfrak{F}$-matrix ring, such that $A^{-1} X A=B^{-1} X B$ for all $X \in \mathfrak{M}$, i.e., we have either $U^{\sigma}=\psi\left(B^{-1} U^{*} B\right)$ or $U^{\sigma}=-\psi\left(B^{-1} U^{* \prime} B\right)$, where $B \in \mathbb{E}(\mathfrak{B})$ and where $U^{*} \in \mathfrak{M}$ with $\psi\left(U^{*}\right)=U$. Conversely, if $B$ is an arbitrary nonsingular $\mathfrak{F}$-matrix, each of the mappings $U \rightarrow \psi\left(B^{-1} U^{*} B\right)$ and $U \rightarrow-\psi\left(B^{-1} U^{* \prime} B\right)$ is a well-defined automorphism of \&. If $U=\psi\left(B^{-1} U^{*} B\right)$ for all $U \in \mathbb{R}$, we have in particular $B^{-1} E_{i j} B=E_{i j}+\lambda I, \lambda \in \mathfrak{F}$, as before, and $\lambda=0$ by the nilpotency of $E_{i j}$ for $i \neq j$, so that $B^{-1} E_{i j} B=E_{i j}$ for all $i \neq j$; thus $B$ is scalar. If $U=-\psi\left(B^{-1} U^{* \prime} B\right)$ for all $U \in \mathbb{R}$, we have $B^{-1} E_{i j} B=\lambda I-E_{j i}$ for all $i \neq j$ as above, and this is impossible as before. Thus the automorphism group $\mathfrak{A}$ of $\mathfrak{R}$ is the product of the group generated by the negative-transpose automorphism $U \rightarrow-\psi\left(U^{* \prime}\right)$ by the projective linear group $P L(\mathfrak{B})$.

Since each $E_{\alpha}^{[p]}=0$ in $L$, we have $\left(E_{\alpha}^{*}\right)^{p}=\lambda I, \lambda \in \mathfrak{F}$, hence $\left(E_{\alpha}^{*}-\lambda^{p^{-1}} I\right)^{p}=0$ in $\mathfrak{M}_{\Omega}$. However, since $E_{\alpha}$ is a root-vector in a standard Cartan decomposition of $\mathcal{R}_{\Omega}$, it follows by our results in the algebraically closed case that $\left(E_{\alpha}^{*}-\lambda^{p^{-1}} I\right)^{7}$ $=0$ in $\mathfrak{M}_{\Re}$. Now $E_{\alpha}^{*}$ is an $\mathfrak{F}$-matrix; thus $\lambda^{p^{-1}}$ satisfies a polynomial equation of degree 7 (and perhaps some equations of lower degree) over $\mathfrak{F}$. But since $\lambda \in \mathfrak{F}$, we know that either $\xi^{p}-\lambda$ is irreducible in the polynomial ring $\mathfrak{F}[\xi]$, therefore is the minimal polynomial of $\lambda^{p^{-1}}$ over $\mathfrak{F}$, or else $\lambda^{p^{-1}} \in \mathfrak{F}$. Since we 
have assumed $p>7$, the polynomial $\xi^{p}-\lambda$ cannot be the minimal polynomial for $\lambda^{p^{-1}}$, so that $\lambda^{p^{-1}} \in \mathfrak{F}$. Thus $X_{\alpha}=E_{\alpha}^{*}-\lambda^{p^{-1}} I \in \mathfrak{M}$ is a nilpotent representative of the coset $E_{\alpha}$, and we see as before that

$$
U \exp \left(\operatorname{ad} E_{\alpha}\right)=\psi\left(\exp \left(-X_{\alpha}\right) U^{*} \exp \left(X_{\alpha}\right)\right),
$$

where $\psi\left(U^{*}\right)=U \in \mathbb{R}$. The group of invariant automorphisms of $\mathbb{R}$ may thus be thought of as a subgroup of $P S L(\mathfrak{B})$.

Since every automorphism of $\mathfrak{M}$ maps its center onto itself, every automorphism of $\mathfrak{M}$ induces an automorphism of $\mathfrak{R}$, hence is either of the form $X \rightarrow A^{-1} X A$ or of the form $X \rightarrow-A^{-1} X^{\prime} A$; for example, if $\sigma$ is an automorphism of $\mathfrak{M}$, and if $X^{\sigma}+\mathfrak{F} I=A^{-1} X A+\mathfrak{F} I$ for all $X \in \mathfrak{M}$, we have $A^{-1} E_{i j} A$ $=E_{i j}^{\sigma}+\lambda_{i j} I$ for $\lambda_{i j} \in \mathcal{F}$ when $i \neq j$, and for $k \neq i, j$, we have $E_{i j}^{\sigma}=\left[E_{i k}^{\sigma} E_{k j}^{\sigma}\right]$ $=\left[A^{-1} E_{i k} A-\lambda_{i k} I, A^{-1} E_{k j} A-\lambda_{k j} I\right]=\left[A^{-1} E_{i k} A, A^{-1} E_{k j} A\right]=A^{-1} E_{i j} A$, i.e., $\lambda_{i j}=0$. Thus $X^{\sigma}=A^{-1} X A$ for all $X \in \mathfrak{M}$; the other case follows by analogy. Conversely, every automorphism of $\&$ has been seen to be induced by an automorphism of $\mathfrak{M}$, and the identity automorphism in either $\mathfrak{Z}$ or $\mathfrak{M}$ occurs only as the first type, and then only when $A$ is scalar. If $\mathfrak{S}$ is the "diagonal" Cartan subalgebra of $\mathfrak{R}$ relative to the basis $e_{1}, \cdots, e_{n}$ for $\mathfrak{B}$, and if $\mathfrak{W}_{0}$ is a second standard Cartan subalgebra, we may deduce as before that there is a nonsingular $A \in \mathbb{E}(\mathfrak{B})$ such that $\psi\left(A^{-1} H^{*} A\right) \in \mathfrak{S}_{0}$ for every $H^{*} \in \mathfrak{M}$ with $\psi\left(H^{*}\right)$ $\in \mathfrak{S}$. Now $\mathfrak{S}$ is the image under $\psi$ of the diagonal subalgebra of $\mathfrak{M}$ relative to $e_{1}, \cdots, e_{n}$, so that the preimage $\psi^{-1}\left(\mathfrak{S}_{0}\right)$ is the diagonal subalgebra of $\mathfrak{M}$ relative to $e_{1} A, \cdots, e_{n} A$. Thus the cosets $\psi\left(\lambda E_{i j}\right), i \neq j, \lambda \in \mathfrak{F}$, where the $E_{i j}$ are the matrix units relative to $e_{1} A, \cdots, e_{n} A$, form the totality of rootvectors of $\mathbb{R}$ relative to $\mathfrak{S}_{0}$. For all $U \in \mathbb{R}$,

$$
U \exp \left(\operatorname{ad}\left(\psi\left(\lambda E_{i j}\right)\right)\right)=\psi\left(\left(\exp \left(\lambda E_{i j}\right)\right)^{-1} U^{*}\left(\exp \left(\lambda E_{i j}\right)\right)\right) .
$$

Chevalley's group $G^{\prime}$ is that generated by the $\exp \left(\operatorname{ad}\left(\lambda E_{i j}\right)\right)$, acting in $\mathfrak{M}$, and is isomorphic to the quotient by its scalar matrices of the group generated by the $\exp \left(\lambda E_{i j}\right)=I+\lambda E_{i j}$. We have observed before that the latter is the unimodular group $S L(\mathfrak{B})$, so that the group $G^{\prime}$ is isomorphic to $P S L(\mathfrak{B})$ in this case as well (see also Ree [19]).

We summarize our results on algebras of type $A$ :

THEOREM 1. If $\mathfrak{R}$ is the Lie algebra of $n$ by $n$ matrices of trace zero over a field $\mathfrak{F}$ of characteristic $\neq 2,3$, whose characteristic does not divide $n(n>1)$, then every automorphism of $\mathfrak{Z}$ is of the form $X \rightarrow A^{-1} X A$ or of the form $X \rightarrow-A^{-1} X^{\prime} A, A$ nonsingular over $\mathfrak{F}$. If $n=2$, all automorphisms are of the former type; if $n>2$, this is not the case, and the automorphism group is the product of $P L(n)$ by the group of order two generated by $X \rightarrow-X^{\prime}$. The group of invariant automorphisms is the group of all automorphisms $X \rightarrow U^{-1} X U$, where $U$ is unimodular, and thus is isomorphic to the group $P S L(n)$.

If $\mathfrak{F}$ is of prime characteristic $p>11$, if $\mathfrak{M}$ is the Lie algebra of $n$ by $n$ 
$\mathfrak{F}$-matrices of trace zero where $p \mid n$, and if $\mathbb{R}$ is the quotient of $\mathfrak{M}$ by the scalar matrices, then every automorphism of $\mathfrak{M}$ is of the form $X \rightarrow A^{-1} X A$ or of the form $X \rightarrow-A^{-1} X^{\prime} A, A$ nonsingular over $\mathfrak{F}$. Every automorphism of $\&$ is of the form $\psi(X) \rightarrow \psi\left(A^{-1} X A\right)$ or of the form $\psi(X) \rightarrow-\psi\left(A^{-1} X^{\prime} A\right), A$ as above, where $\psi$ is the canonical homomorphism of $\mathfrak{M}$ onto $\mathfrak{R}$. The groups of automorphisms of $\mathfrak{R}$ and of $\mathfrak{M}$ are both isomorphic in this fashion to the product of $\operatorname{PSL}(n)$ by a group of order 2 . The group of invariant automorphisms of $\mathfrak{R}$ consists of the mappings $\psi(X) \rightarrow \psi\left(U^{-1} X U\right), U \in S L(n)$, and is thus isomorphic to $\operatorname{PSL}(n)$.

The group $G^{\prime}$ of Chevalley formed over $\mathfrak{F}$ from a complex Lie algebra of type $A_{n-1}$ is the group of invariant automorphisms of the corresponding algebra $\mathfrak{R}$ above, and is isomorphic to the projective unimodular group $\operatorname{PSL}(n, \mathfrak{F})$.

3. Algebras of type $C$. Let $\mathfrak{B}$ be a $2 n$-dimensional vector space over a field $\mathfrak{F}$ of characteristic not 2 or 3 , and assume that $\mathfrak{B}$ carries a nondegenerate alternate bilinear form $(x, y)$ over $\mathfrak{F}$. Assume further that $n>1$. Let $\&$ be the Lie algebra of all elements $T \in \mathbb{E}(\mathfrak{B})$ satisfying $(x T, y)+(x, y T)=0$ for all $x, y \in \mathfrak{B}$. If $\mathfrak{F}$ is of prime characteristic $p$, then $T^{p} \in \mathbb{R}$ if $T \in \mathfrak{R}$; thus $\mathfrak{R}$ is a restricted Lie algebra under $p$ th iterates. (This is a special case of the more general fact that the algebra $\subseteq$ of (6) is restricted, as is seen from (10) by setting $k=p$.) By displaying a suitably chosen basis for $\mathfrak{Z}[12 ; 14 ; 20]$, one can see that $\mathbb{R}$ is a Lie algebra of classical type $C_{n}$, hence is (normal) simple $[18$, Theorem 8]. Further observation of this basis shows that $\mathbb{E}(\mathfrak{B})=\mathfrak{F} I+\mathbb{R}$ $+\mathfrak{Z}^{2}$. For each root-vector $E_{\alpha}$ relative to a standard Cartan subalgebra of $\mathbb{R}$, we have $\left(\operatorname{ad} E_{\alpha}\right)^{3}=0$. It follows by Lemma 1 and by the nilpotency of $E_{\alpha}$ (which is proved as in $\S 2$ ) that $E_{\alpha}^{5}=0$, hence that for characteristics $p \neq 5,7$ we have $X \exp \left(\operatorname{ad} E_{\alpha}\right)=\left(\exp \left(-E_{\alpha}\right)\right) X\left(\exp E_{\alpha}\right)$, where $A=\exp \left(E_{\alpha}\right)$ satisfies $(x A, y A)=(x, y)$ for all $x, y \in \mathfrak{B}$. The latter observations result from (1), (2) and $(7)$. Moreover, $\operatorname{det} A=1$. Since $\&(\mathfrak{B})$ is generated by $\mathbb{R}$, we have $A^{-1} X A$ $=X$ for all $X \in \mathbb{R}$ if and only if $A=\lambda I, \lambda \in \mathfrak{F}$. The linear transformations $A$ of $\mathfrak{B}$ satisfying $(x A, y A)=(x, y)$ for all $x, y \in \mathfrak{B}$ constitute the symplectic group $S p(\mathfrak{B})$. Thus we see that the group $\Im$ of invariant automorphisms of $\mathfrak{R}$ is isomorphically embedded in the projective symplectic group $P S p(\mathfrak{B})$, i.e. the quotient of $P S p(\mathfrak{B})$ by the scalar matrices in it. Since each mapping $X \rightarrow A^{-1} X A, A \in S p(\mathfrak{B})$, is an automorphism of $\mathfrak{l}$, we see that the group $P S p(\mathfrak{B})$ may in turn be regarded as a subgroup of the full automorphism group $\mathfrak{A}$ of $\mathbb{R}$.

If $\mathfrak{F}$ is algebraically closed, we have seen in (A) that $\Im$ is the full automorphism group $\mathfrak{A}$. In the general case, let $\Omega$ be the algebraic closure of $\mathfrak{F}$. Then $\Omega_{\Omega}$ is readily seen to be the Lie algebra of all linear transformations $T$ of $\mathfrak{B}_{\Re}$ satisfying $(x T, y)+(x, y T)=0$ for all $x, y \in \mathfrak{B}_{\Omega}$, where the alternate bilinear form $(x, y)$ on $\mathfrak{B}_{\Omega}$ is the unique $\Omega$-bilinear extension of the original alternate form $(x, y)$ on $\mathfrak{B}$. If $\sigma$ is an automorphism of $\mathfrak{R}$, then $\sigma$ admits a unique extension to an automorphism, also denoted by $\sigma$, of $\mathfrak{R}_{\Omega}$. Thus there 
is an $S \in S p\left(\mathfrak{B}_{\Omega}\right)$ such that $X^{\sigma}=A^{-1} X A$ for all $X \in \Omega_{\Omega}$, by the earlier result that $\Im=P S p\left(\mathfrak{B}_{\Re}\right)=\mathfrak{A}$ for $\mathfrak{R}_{\Omega}$. In particular, $X^{\sigma}=A^{-1} X A$ for all $X \in \mathbb{R}$. It follows by the result of [25] cited in $\$ 2$ that there is a nonsingular $B \in \mathbb{E}(\mathfrak{B})$ such that $X^{\sigma}=B^{-1} X B$ for all $X \in \mathcal{R}$, and thus we have $X^{\sigma}=B^{-1} X B$ for all $X \in \Omega_{\Omega}$, by the linearity over $\Omega$ of the mapping $X \rightarrow B^{-1} X B$, which coincides with $\sigma$ on a basis for $\mathfrak{Q}_{\mathfrak{R}}$. Therefore $B=\lambda A, \lambda \in \Omega$, and for $x, y \in \mathfrak{B}$ we have $(x B, y B)=(\lambda x A, \lambda y A)=\lambda^{2}(x A, y A)=\lambda^{2}(x, y)$, i.e., $(x B, y B)=(x, y) \beta$, where $0 \neq \beta \in \mathfrak{F}$, for all $x, y \in \mathfrak{B}$. Conversely, if $B$ is any linear transformation of $\mathfrak{B}$ such that for some nonzero $\beta$ in $\mathfrak{F},(x B, y B)=(x, y) \beta$ for all $x, y \in \mathfrak{B}$, then the mapping $X \rightarrow B^{-1} X B$ is readily seen to be an automorphism of $\mathbb{R}$. The totality of such mappings is a group (S); thus the quotient $B^{*} / \mathfrak{F}^{*} I$ of $B$ by the scalar transformations in $B S$ is mapped isomorphically onto the full automorphism group $\mathfrak{A}$ of $\mathfrak{R}$.

Let $\mathfrak{S}$ be the diagonal Cartan subalgebra of $\mathfrak{R}$ displayed in matrix form in $[12 ; 14 ; 20]$ relative to a basis $e_{1}, \cdots, e_{2 n}$ for $\mathfrak{B}$ such that $\left(e_{i}, e_{i+n}\right)=1$ $=-\left(e_{i+n}, e_{i}\right), 1 \leqq i \leqq n$, all other $\left(e_{j}, e_{k}\right)=0$. If $\mathfrak{S}_{0}$ is a second standard Cartan subalgebra of $\mathbb{R}$, it follows by the absence of isomorphisms with algebras of different types $[12 ; 14 ; 21]$ and by the isomorphism theorem of $[18]$ that there is an automorphism $\sigma$ of $\mathbb{R}$ such that $\mathfrak{S}^{\sigma}=\mathfrak{S}_{0}$. That is, there is a $B$ in $\mathfrak{E}(\mathfrak{B})$ with $(x B, y B)=(x, y) \beta$ for a fixed $\beta \neq 0$ in $\mathfrak{F}$, and for all $x, y \in \mathfrak{B}$, and such that $B^{-1} \mathfrak{S}_{E} B=\mathfrak{S}_{0}$, or $\mathfrak{S}_{0}$ is the set of diagonal matrices in $\mathbb{R}$ with respect to the basis $e_{1} B, \cdots, e_{2 n} B$ for $\mathfrak{B}$. Now $\left(e_{j} B, e_{k} B\right)=\left(e_{j}, e_{k}\right) \beta$ for $1 \leqq j, k \leqq 2 n$. The system of root-vectors in $\mathbb{Q}$ relative to $\mathfrak{F}$ consisting of those transformations of $\mathfrak{B}$ whose matrices relative to $e_{1}, \cdots, e_{2 n}$ are the $E_{i j}-E_{j+n, i+n}$; $E_{i, j+n}+E_{j, i+n} ; E_{i+n, j}+E_{j+n, i} ; E_{i, i+n} ; E_{i+n, i} ; 1 \leqq i, j \leqq n, i \neq j$, (see $[12 ; 14 ; 20]$ ) is transformed into the system $\left\{E_{\alpha}\right\}$ of transformations whose matrices relative to $e_{1} B, \cdots, e_{2 n} B$ constitute the same set of combinations of matrix units as the above. Chevalley's group $G^{\prime}$ formed over $\mathfrak{F}$ from a complex Lie algebra of type $C_{n}$ is then the group of automorphisms of $\mathbb{\&}$ generated by the $\exp \left(\operatorname{ad}\left(\lambda E_{\alpha}\right)\right)$ for all $\lambda \in \mathfrak{F}$, where $E_{\alpha}$ runs through this set. To show that the group $\Im$ of invariant automorphisms is $P S p(\mathfrak{B})$, it will suffice to show that $G^{\prime}=P S p(\mathfrak{B})$, and this will follow if one knows that the corresponding transformations $\exp \left(\lambda E_{\alpha}\right)$ generate $S p(\mathfrak{B})$. That is, we have a basis $u_{1}, \cdots, u_{2 n}$ for $\mathfrak{B}$ such that $\left(u_{i}, u_{i+n}\right)=\beta=-\left(u_{i+n}, u_{i}\right), 0 \neq \beta \in \mathfrak{F}$, and such that $\left(u_{j}, u_{k}\right)=0$ otherwise. Since each $E_{\alpha}^{2}=0$, the exponentials are simply $I+\lambda\left(E_{i j}-E_{j+n, i+n}\right)$; $I+\lambda\left(E_{i, j+n}+E_{j, i+n}\right) ; I+\lambda\left(E_{i+n, j}+E_{j+n, i}\right) ; I+\lambda E_{i, i+n} ; I+\lambda E_{i+n, i}$. Here the indices are as before and the $E_{j k}$ are the matrix-unit transformations relative to the $u_{i}$. The corresponding proof by Dickson [7] cited by Ree [19] can be modified trivially to show that $S p(\mathfrak{B})$ is generated by transformations of these types. Thus the assertion $G^{\prime}=\mathfrak{Y}=P S p(\mathfrak{B})$ follows. Again to summarize:

THEOREM 2. If $\mathbb{R}$ is the Lie algebra of skew transformations of a vector space $\mathfrak{B}$ of finite dimension $>2$ carrying a nondegenerate alternate bilinear form $(x, y)$ over a field $\mathfrak{F}$ of characteristic $\neq 2,3,5,7$, then every automorphism of $\mathfrak{R}$ is of the 
form $X \rightarrow A^{-1} X A$, where $A$ is a linear transformation of $\mathfrak{B}$ satisfying $(x A, y A)$ $=(x, y) \alpha$ for all $x, y \in \mathfrak{B}$ and for some fixed $\alpha \neq 0$ in $\mathfrak{F}$. Thus the automorphism group $\mathfrak{A}$ of $\mathfrak{Z}$ is isomorphic to the quotient group of the group (f) of all such transformations $A$ of $\mathfrak{B}$ by the group of nonzero scalar transformations. The group $\Im$ of invariant automorphisms is the group of all automorphisms of the form $X \rightarrow U^{-1} X U$, where $(x U, y U)=(x, y)$ for all $x, y \in \mathfrak{B}$, thus is isomorphic with the projective symplectic group $P S p(\mathfrak{B})$. The group $G^{\prime}$ of Chevalley formed over $\mathfrak{F}$ from a complex Lie algebra of type $C_{n}$ coincides with $\Im$, and is thereby isomorphic with $P S p(\mathfrak{B})$.

4. Algebras of type $B$. Let $\mathfrak{B}$ be a vector space of dimension $2 n+1(n \geqq 1)$ over a field $\mathfrak{F}$ of characteristic different from 2 , and let $(x, y)$ be a symmetric nondegenerate bilinear form of maximal Witt index on $\mathfrak{B}$; that is, $\mathfrak{B}$ contains an $n$-dimensional subspace $\mathfrak{U}$ on which the form is identically zero. We further assume that the discriminant $\Delta$ of the form is a square in $\mathfrak{F}$ if $n$ is even, and that $-\Delta$ is a square in $\mathfrak{F}$ if $n$ is odd. Then it follows that a basis $e_{1}, \cdots$, $e_{2 n+1}$ can be chosen for $\mathfrak{B}$ over $\mathfrak{F}$ satisfying $\left(e_{1}, e_{1}\right)=1 ;\left(e_{j}, e_{n+j}\right)=1=\left(e_{n+j}, e_{j}\right)$, $2 \leqq j \leqq n+1$; all other $\left(e_{i}, e_{k}\right)=0$. Let $\mathfrak{R}$ be the Lie algebra of all $T \in \mathbb{E}(\mathfrak{B})$ satisfying $(x T, y)+(x, y T)=0$ for all $x, y \in \mathfrak{B}$. Then examination of a suitably chosen basis for $\mathfrak{\&}$ (about which more will be said later; see also $[12 ; 14 ; 20]$ ) reveals that $\mathfrak{R}$ is a Lie algebra of classical type $B_{n}$ if the characteristic of $\mathfrak{F}$ is not 2 or 3 , hence is simple. Exactly as in $\S 3$, we conclude that the relevant formulas of $\$ 1$ involving exponentials are valid if the characteristic of $\mathfrak{F}$ is not $2,3,5,7$. We assume this in the sequel.

For all $X \in \mathcal{R}$, we have $X \exp \left(\operatorname{ad} E_{\alpha}\right)=\left(\exp E_{\alpha}\right)^{-1} X\left(\exp E_{\alpha}\right)$, and $A=\exp E_{\alpha}$ is unimodular and satisfies $(x A, y A)=(x, y)$ for all $x, y \in \mathfrak{B}$ by $(7)$. Thus every invariant automorphism is of the form $X \rightarrow A^{-1} X A$ where $A$ is in the rotation group $O^{+}(\mathfrak{B})$; the only scalar transformation in $O^{+}(\mathfrak{B})$ is the identity, so the group of invariant automorphisms may be regarded as a subgroup of $O^{+}(\mathfrak{B})$ (again $\mathfrak{E}(\mathfrak{B})=\mathfrak{F} I+\mathfrak{L}+\mathfrak{R}^{2}$ ). For each $A \in O^{+}(\mathfrak{B})$, the mapping $X \rightarrow A^{-1} X A$ is an automorphism of $\mathfrak{l}$, so $O^{+}(\mathfrak{B})$ may be regarded as a subgroup of the full automorphism group $\mathfrak{A}$ of $\mathfrak{R}$.

In the algebraically closed case, we have seen in (A) that $\mathfrak{Y}=\mathfrak{A}$; hence we have $\mathfrak{F}=O^{+}(\mathfrak{B})=\mathfrak{A}$ when $\mathfrak{F}$ is algebraically closed. If we now drop the assumption that $\mathfrak{F}$ is algebraically closed and let $\Re$ be its algebraic closure, we see as in previous cases that $\ell_{\Omega}$ is the Lie algebra of skew transformations in $\mathfrak{B}_{\Re}$ with respect to the $\Re$-bilinear form determined by $(x, y)$, and, by the reasoning of $\S 3$, that every automorphism of $\mathfrak{l}$ is of the form $X \rightarrow A^{-1} X A$, where $A \in \mathfrak{F}(\mathfrak{B})$ has the property that for some $\alpha \neq 0$ in $\mathfrak{F}$ and for all $x, y \in \mathfrak{B}$, $(x A, y A)=(x, y) \alpha$. If $\mathbb{B}$ denotes the group of all $A$ of this form, then $\mathbb{B}$ contains $\mathfrak{F}^{*} I$ as before, and as in $\S 3$ the full automorphism group $\mathfrak{A}$ of $\mathfrak{R}$ is isomorphic to $\mathfrak{S} / \mathfrak{F}^{*} I$ via the obvious mapping of the latter group onto $\mathfrak{A}$. It remains to consider the group of invariant automorphisms of $\mathbb{R}$.

The transformations in $\mathfrak{R}$ whose matrices relative to the basis $e_{1}, \cdots, e_{2 n+1}$ 
of $\mathfrak{B}$ are diagonal constitute a standard Cartan subalgebrå $\mathfrak{E}$, and each rootspace relative to $\mathfrak{S}$ is spanned by a transformation expressible in terms of matrix units as: $E_{i j}-E_{j+n, i+n} ; E_{i, j+n}-E_{j, i+n} ; E_{i+n, j}-E_{j+n, i} ; E_{1 j}-E_{j+n, 1}$; $E_{j 1}-E_{1, j+n} ; 2 \leqq i, j \leqq n+1, i \neq j$. If $\mathfrak{S}_{0}$ is a second standard Cartan subalgebra, then as before there is an automorphism of $\mathfrak{R}$ mapping $\mathfrak{S}$ onto $\mathfrak{S}_{0}$, i.e., an $A \in \mathbb{B}$ with $A^{-1} \mathfrak{S} A=\mathfrak{S}_{0}$. If $e_{1} A, \cdots, e_{2 n+1} A$ is the transformed basis for $\mathfrak{B}$, then $\left(e_{j} A, e_{k} A\right)=\left(e_{j}, e_{k}\right) \alpha$ for all $j, k$ and for some fixed nonzero $\alpha \in \mathfrak{F}$. Each root-space relative to $\mathfrak{S}_{0}$ is spanned by an $E_{\delta}$ whose matrix relative to $e_{1} A, \cdots, e_{2 n+1} A$ is one of those listed above. Thus we have a basis $u_{1}, \cdots, u_{2 n+1}$ for $\mathfrak{B}$ such that $\left(u_{1}, u_{1}\right)=\alpha=\left(u_{j}, u_{n+j}\right)=\left(u_{n+j}, u_{j}\right), 2 \leqq j \leqq n+1$, all other $\left(u_{i}, u_{k}\right)=0$; we consider the group of linear transformations of $\mathfrak{B}$ generated by the exponentials of root-vectors relative to $\mathfrak{S}_{0}$, or by the transformations whose matrices relative to this basis are of the forms

$$
\begin{aligned}
& I+\lambda\left(E_{i j}-E_{j+n, i+n}\right) ; I+\lambda\left(E_{i, j+n}-E_{j, i+n}\right) ; I+\lambda\left(E_{i+n, j}-E_{j+n, i}\right) ; \\
& I+\lambda\left(E_{1 j}-E_{j+n, 1}\right)-2^{-1} \lambda^{2} E_{j+n, j} ; I+\lambda\left(E_{j 1}-E_{1, j+n}\right)-2^{-1} \lambda^{2} E_{j, j+n} ; \\
& 2 \leqq i, j \leqq n+1, i \neq j .
\end{aligned}
$$

This group is a subgroup of $\Im$, which is in turn a subgroup of $O^{+}(\mathfrak{B})$. By utilizing the procedure of Theorem 3.20 of [1] and the spinorial norm mapping of $O^{+}(\mathfrak{B})$ into $\mathfrak{F}^{*} /\left(\mathfrak{F}^{*}\right)^{2}$ found in Chapter 5 of [1] (since $\mathfrak{B}$ contains isotropic vectors) one sees that each of the above transformations is in the commutator subgroup $\Omega(\mathfrak{B})$ of the full orthogonal group $O(\mathfrak{B})$. Since $\mathfrak{S}_{0}$ is an arbitrary standard Cartan subalgebra, it follows that $\Im \subseteq \Omega(\mathfrak{B})$. It does not seem to be an immediate consequence of the work of Dickson [7] and its utilization by Ree [19] under somewhat different conditions that the transformations (15) generate $\Omega(\mathfrak{B})$. This is in fact the case. If $\mathfrak{B}$ has dimension greater than 3 , then we may post-multiply any $T \in O(\mathfrak{B})$ by a sequence of transformations of the types (15) to obtain a transformation $U \equiv T$ (modulo products of (15)) with $u_{2} U=u_{2}, u_{n+2} U=u_{n+2}$, hence mapping the subspace $\mathfrak{u}$ spanned by $u_{1}, u_{3}, \cdots, u_{n+1}, u_{n+3}, \cdots, u_{2 n+1}$ into itself. (The method of choice of these transformations from (15) is more or less explicitly indicated by the corresponding argument in $\S 6$.) If $T \in \Omega(\mathfrak{B})$, so is $U$, and the spinorial norm of the restriction of $U$ to $\mathfrak{u}$ is the same as that of $U$. Hence $\dot{U} \mid \mathfrak{u} \in \Omega(\mathfrak{u})$. If we make the inductive assumption that our assertion holds for $\mathfrak{U}$ and observe that the generators of the type $(15)$ for $\Omega(\mathfrak{U})$ are the restrictions to $\mathfrak{u}$ of generators (15) above which map $u_{2}$ onto $u_{2}$ and $u_{n+2}$ onto $u_{n+2}$, we see that $U$ is in the group generated by the set (15). Our proof by induction will then be completed if we carry out the proof for $\operatorname{dim} \mathfrak{B}=3$. Here if $T \in O^{+}(\mathfrak{B})$, the kind of reduction initially carried out above shows that $T \equiv U$ (modulo products of (15)), where $U=\operatorname{diag}\left\{1, \lambda, \lambda^{-1}\right\}$. The spinorial norm of $U$ is the identity, i.e., $U \in \Omega(\mathfrak{B})$, if and only if $\lambda \in \mathfrak{F}^{* 2}$. Thus if $T \in \Omega(\mathfrak{B})$, we have $U=\operatorname{diag}\left\{1, \delta^{2}, \delta^{-2}\right\}$. But then 


$$
\begin{aligned}
U= & \left(I+\lambda_{1}\left(E_{12}-E_{31}\right)-2^{-1} \lambda_{1}^{2} E_{32}\right) \cdot\left(I+\lambda_{2}\left(E_{21}-E_{13}\right)-2^{-1} \lambda_{2}^{2} E_{23}\right) \\
& \cdot\left(I+\lambda_{3}\left(E_{12}-E_{31}\right)-2^{-1} \lambda_{3}^{2} E_{32}\right) \cdot\left(I+\lambda_{4}\left(E_{21}-E_{13}\right)-2^{-1} \lambda_{4}^{2} E_{23}\right),
\end{aligned}
$$

where $\lambda_{1}=2 \delta^{-1}\left(\delta^{-1}-1\right), \lambda_{2}=1, \lambda_{3}=2\left(\delta^{-1}-1\right), \lambda_{4}=-\delta$, so that $U$ is in the group generated by the set (15). Thus the group generated by these transformations is $\Omega(\mathfrak{B})$ in all cases and we have $\mathfrak{Y}=\Omega(\mathfrak{B})$. To summarize:

THEOREM 3. If $\mathbb{R}$ is the Lie algebra of skew transformations of a vector space $\mathfrak{B}$ of odd dimension $2 n+1 \geqq 3$, carrying a nondegenerate symmetric bilinear form of maximal index and satisfying the discriminant condition $(-1)^{n} \Delta \in \mathfrak{F}^{2}$, over a field $\mathfrak{F}$ of characteristic $\neq 2,3,5,7$, then every automorphism of $\mathbb{R}$ is of the form $X \rightarrow A^{-1} X A$, where $A$ is a linear transformation of $\mathfrak{B}$ satisfying $(x A, y A)$ $=(x, y) \alpha$ for all $x, y \in \mathfrak{B}$ and for some $\alpha \neq 0$ in $\mathfrak{F}$. The automorphism group $\mathfrak{A}$ of $\mathfrak{R}$ is isomorphic to the quotient group of the group \&s of all such similitudes $A$ by the nonzero scalar transformations. The group $\Im$ of invariant automorphisms is the group of all automorphisms $X \rightarrow U^{-1} X U$, where $U$ is in the commutator subgroup $\Omega(\mathfrak{B})$ of the orthogonal group $O(\mathfrak{B})$; thus $\Im$ is isomorphic with $\Omega(\mathfrak{B})$. The group $G^{\prime}$ of Chevalley formed over $\mathfrak{F}$ from a complex Lie algebra of type $B_{n}$ is the full group $\Im$.

5. Algebras of type $D$. Let $\mathfrak{B}$ be a vector space of dimension $2 n(n \geqq 3)$ over a field $\mathfrak{F}$ of characteristic $\neq 2$, and let $(x, y)$ be a symmetric nondegenerate bilinear form of maximal Witt index on $\mathfrak{B}$. Then a basis $e_{1}, \cdots, e_{2 n}$ for $\mathfrak{B}$ over $\mathfrak{F}$ can be chosen with $\left(e_{i}, e_{i+n}\right)=1=\left(e_{i+n}, e_{i}\right), 1 \leqq i \leqq n,\left(e_{j}, e_{k}\right)=0$ otherwise. Let $\mathfrak{R}$ be the Lie algebra of all $T \in \mathfrak{E}(\mathfrak{B})$ satisfying $(x T, y)+(x, y T)=0$ for all $x, y \in \mathfrak{B}$. As before, the basis for $\mathfrak{R}$ displayed in $[12 ; 14 ; 20]$ shows that $\mathfrak{R}$ is a simple Lie algebra of classical type $D_{n}$ if the characteristic of $\mathfrak{F}$ is not 2 or 3 (for $n=3$, there is no distinction between type $D_{3}$ and type $A_{3}$ ), that $\mathfrak{E}(\mathfrak{B})=\mathfrak{F} I+\mathfrak{R}+\mathfrak{R}^{2}$, and, as in $\S \S 3,4$, that $E_{\alpha}^{5}=0$ for all root-vectors $E_{\alpha}$ relative to standard Cartan subalgebras. Thus we assume further that the characteristic of $\mathfrak{F}$ is not $2,3,5,7$.

As in type $B$, every invariant automorphism of $\mathbb{R}$ is of the form $X \rightarrow A^{-1} X A$, where $A$ is in the rotation group $O^{+}(\mathfrak{B})$. Each $A$ in $O^{+}(\mathfrak{B})$ induces an automorphism of $\mathfrak{R}$ by the above mapping, and the identity is induced if and only if $A$ is scalar, i.e., $A= \pm I$. Thus we may regard the group $\Im$ of invariant automorphisms as a subgroup of the projective rotation group $\mathrm{PO}^{+}(\mathfrak{B})$.

Next let $U$ be the element of $E(\mathfrak{B})$ whose matrix relative to the basis $\left\{e_{i}\right\}$ is $I-E_{n, n}-E_{2 n, 2 n}-E_{n, 2 n}-E_{2 n, n}$. Then it is readily checked that $\left(e_{i} U, e_{j} U\right)$ $=\left(e_{i}, e_{j}\right)$ for all $i, j$, so that $U \in O(\mathfrak{B})$, the orthogonal group; moreover, $U$ has determinant -1 , so is not in $O^{+}(\mathfrak{B})$. The mapping $X \rightarrow U^{-1} X U$ is an automorphism of $\mathfrak{R}$ of period two. If $U^{-1} X U=A^{-1} X A$ for all $X \in \mathfrak{R}$, where $A \in O^{+}(\mathfrak{B})$, then we have $U=\lambda A$, where $\lambda \in \mathfrak{F}$, and so $\lambda I \in O(\mathfrak{B})$, with $\operatorname{det}(\lambda I)$ $=-1$. But since $\lambda I \in O(\mathfrak{B}), \lambda= \pm 1$, and $\operatorname{det}(\lambda I)=1$, a contradiction. Hence the mapping $\sigma: X \rightarrow U^{-1} X U$ is not in $\Im$, and $\Im$ is not the full automorphism group $\mathfrak{A}$. 
Suppose now that $n=4$; the basis for $\mathfrak{R}$ referred to above contains a basis for the Cartan subalgebra $\mathfrak{S}$ consisting of elements whose matrices relative to the basis $e_{1}, \cdots, e_{8}$ are diagonal, and contains a system of root-vectors relative to $\mathfrak{S}$ consisting of elements whose matrices relative to this basis are $E_{i j}-E_{j+4_{2} i+4}, i \neq j ; E_{i, j+4}-E_{j, i+4}, i<j ; E_{i+4, j}-E_{j+4, i}, i<j ; 1 \leqq i, j \leqq 4$. If we set $E_{\alpha_{1}}=E_{21}-E_{56}, E_{\alpha_{2}}=E_{32}-E_{67}, E_{\alpha_{3}}=E_{43}-E_{78}, E_{\alpha_{4}}=E_{74}-E_{83}$, then $\alpha_{1}, \alpha_{2}$, $\alpha_{3}, \alpha_{4}$ form a fundamental system of roots of type $D_{4}$ relative to $\mathfrak{E}$ with the Cartan integers $A_{i 2}=A_{2 i}=-1, i=1,3,4 ; A_{j j}=2,1 \leqq j \leqq 4 ; A_{i k}=0$ if $i \neq k, i$ and $k$ among 1, 3, 4. Thus by Theorem 9 of [18] and Lemma 6 of (A), there is a unique automorphism $\phi$ of $\mathfrak{R}$ mapping $E_{\alpha_{1}}$ onto $E_{\alpha_{3}}, E_{\alpha_{3}}$ onto $E_{\alpha_{4}}$, $E_{\alpha_{1}}$ onto $E_{\alpha_{1}}, E_{\alpha_{2}}$ onto $E_{\alpha_{2}}$, and $\mathfrak{S}$ onto $\mathfrak{S}$, and $\phi$ is of period 3 . If we set $E_{-\alpha_{1}}=E_{12}-E_{65}, E_{-\alpha_{2}}=E_{23}-E_{76}, E_{-\alpha_{3}}=E_{34}-E_{87}, E_{-\alpha_{4}}=E_{47}-E_{38}$, then each $\left[E_{-\alpha_{i}} E_{\alpha_{i}}\right] \in \mathfrak{G}$, and $\alpha_{i}\left(\left[E_{-\alpha_{i}} E_{\alpha_{i}}\right]\right)=2,1 \leqq i \leqq 4$. By the proof of Lemma 6 of (A), $\phi$ sends $E_{-\alpha_{1}}$ onto $E_{-\alpha_{3}}, E_{-\alpha_{3}}$ onto $E_{-\alpha_{4}}, E_{-\alpha_{4}}$ on to $E_{-\alpha_{1}}, E_{-\alpha_{2}}$ onto $E_{-\alpha_{2}}$. Now we can give an explicit expression for $\phi$ by using the fact that the $E_{\alpha_{i}}$ and $E_{-\alpha_{i}}$ generate $\mathfrak{R}$. The general element of $\mathfrak{R}$ has a matrix of the form

$$
\left(\frac{(\xi)}{(\zeta)} \mid \frac{(\eta)}{-(\xi)^{\prime}}\right)
$$

where $(\xi),(\eta),(\zeta)$ are 4 by $4 \mathfrak{F}$-matrices and where $(\eta)^{\prime}=-(\eta),(\zeta)^{\prime}=-(\zeta)$. The result is then that $\phi$ sends this element into

$$
\left(\frac{(\mu)}{(\rho)} \mid \frac{(\nu)}{-(\mu)^{\prime}}\right)
$$

where

$$
\begin{aligned}
& (\mu)=\left(\begin{array}{rrrr}
2^{-1}\left(\xi_{11}+\xi_{22}+\xi_{33}+\xi_{44}\right) & -\eta_{34} & \eta_{24} & -\eta_{14} \\
\zeta_{34} & 2^{-1}\left(\xi_{11}+\xi_{22}-\xi_{33}-\xi_{44}\right) & \xi_{23} & -\xi_{13} \\
-\zeta_{24} & \xi_{32} & 2^{-1}\left(\xi_{11}-\xi_{22}+\xi_{33}-\xi_{44}\right) & \xi_{12} \\
\zeta_{14} & -\xi_{31} & \xi_{21} & 2^{-1}\left(-\xi_{11}+\xi_{22}+\xi_{33}-\xi_{44}\right)
\end{array}\right), \\
& (\nu)=\left(\begin{array}{cccc}
0 & \eta_{12} & \eta_{13} & \eta_{23} \\
-\eta_{12} & 0 & -\xi_{14} & -\xi_{24} \\
-\eta_{13} & \xi_{14} & 0 & -\xi_{34} \\
-\eta_{23} & \xi_{24} & \xi_{34} & 0
\end{array}\right), \quad(\rho)=\left(\begin{array}{cccc}
0 & \zeta_{12} & \zeta_{13} & \zeta_{23} \\
-\zeta_{12} & 0 & \xi_{41} & \xi_{42} \\
-\zeta_{13} & -\xi_{41} & 0 & \xi_{43} \\
-\zeta_{23} & -\xi_{42} & -\xi_{43} & 0
\end{array}\right) .
\end{aligned}
$$

If there were a nonsingular transformation $A$ of $\mathfrak{B}$ such that $X^{\phi}=A^{-1} X A$ for all $X \in \mathbb{R}$, then we should have $A X^{\phi}=X A$ for all $X \in \mathbb{R}$. If $(\alpha)$ is the matrix of $A$ relative to the basis $e_{1}, \cdots, e_{8}$ above, we have in particular $A\left(E_{43}-E_{78}\right)$ $=\left(E_{21}-E_{56}\right) A, A\left(E_{74}-E_{83}\right)=\left(E_{43}-E_{78}\right) A$. The first of these equations implies $\alpha_{i 7}=0, i \neq 2,5$; the second implies $\alpha_{i 7}=0, i \neq 4,7$. Hence all $\alpha_{i 7}=0$, and $A$ cannot be nonsingular.

Note. A more conceptual demonstration of such an "outer" automorphism could be given using the principle of (local) triality $[8 ; 16]$. This prin- 
ciple will be invoked in dealing with the exceptional algebras, but we prefer to avoid it here.

Since $\phi$ is of period $3, \phi^{2}=\phi^{-1}$ is not of the form $X \rightarrow A^{-1} X A$. With $\sigma$ as previously introduced, the effect of $\sigma$ when $n=4$ is to send

$$
\left(\frac{(\xi)}{(\zeta)} \mid \frac{(\eta)}{-(\xi)^{\prime}}\right) \text { into }\left(\frac{(\mu)}{(\rho)} \mid \frac{(\nu)}{-(\mu)^{\prime}}\right)
$$

where

$$
(\mu)=\left(\begin{array}{lll}
\xi_{11} & \xi_{12} & \xi_{13}-\eta_{14} \\
\xi_{21} & \xi_{22} & \xi_{23}-\eta_{24} \\
\xi_{31} & \xi_{32} & \xi_{33}-\eta_{34} \\
\zeta_{14} & \zeta_{24} & \zeta_{34}-\xi_{44}
\end{array}\right), \quad(\nu)=\left(\begin{array}{cccc}
0 & \eta_{12} & \eta_{13}-\xi_{14} \\
-\eta_{12} & 0 & \eta_{23}-\xi_{24} \\
-\eta_{13} & -\eta_{23} & 0 & -\xi_{34} \\
\xi_{14} & \xi_{24} & \xi_{34} & 0
\end{array}\right),
$$

$$
(\rho)=\left(\begin{array}{cccc}
0 & \zeta_{12} & \zeta_{13} & \xi_{41} \\
-\zeta_{12} & 0 & \zeta_{23} & \xi_{42} \\
-\zeta_{13} & -\zeta_{23} & 0 & \xi_{43} \\
-\xi_{41} & -\xi_{42} & -\xi_{43} & 0
\end{array}\right)
$$

From (16) and (17) one readily sees that $\sigma \phi \sigma=\phi^{2}$, so that $\sigma$ and $\phi$ generate a group isomorphic to the symmetric group $\Sigma_{3}$, with elements $\iota, \sigma, \phi, \phi^{2}, \sigma \phi, \sigma \phi^{2}$. The cosets of these automorphisms by the invariant automorphisms are distinct; to see this, since they form a group, it is enough to show that $\iota$ is the only invariant automorphism among them. We have already seen that $\sigma, \phi, \phi^{2}$ are not invariant automorphisms; if $\sigma \phi$ or $\sigma \phi^{2}$ were an invariant automorphism $\psi$, then we should have either $\phi=\sigma \psi$ or $\phi^{2}=\sigma \psi$, an automorphism of the form $X \rightarrow A^{-1} X A$. But this has been seen above to be impossible. Thus the index of the group of invariant automorphisms in the full automorphism group is at least 2 if $n \neq 4$ and at least 6 if $n=4$.

In the algebraically closed case, we have seen in (A) that the index of $\Im$ in $\mathfrak{A}$ is at most 2 if $n \neq 4$ and at most 6 if $n \neq 4$. Hence these values are exact. In every case, the automorphism group $\mathfrak{A}$ contains the quotient $P O(\mathfrak{B})$ of the full orthogonal group by the scalars, which, by virtue of the existence of $\sigma$, contains properly the group $\mathrm{PO}^{+}(\mathfrak{B})$, and this in turn contains $\Im$. Since $[\mathfrak{A}: \mathfrak{Y}]=2$ if $n \neq 4$, we have $\mathfrak{A}=P O(\mathfrak{B}), \mathfrak{S}=P O^{+}(\mathfrak{B})$ if $n \neq 4$. If $n=4$, the cosets of $\iota, \phi, \phi^{2}$ by $P O(\mathfrak{B})$ are distinct, so that $[\mathfrak{A}: P O(\mathfrak{B})] \geqq 3$, $\left[P O(\mathfrak{B}): P O^{+}(\mathfrak{B})\right] \equiv 2$, and $[\mathfrak{A}: \mathfrak{Y}]=6$. It follows that $[\mathfrak{A}: P O(\mathfrak{B})]=3$ and that $\mathfrak{Y}=P O^{+}(\mathfrak{B}) ; \mathfrak{A}$ is the product of $P O(\mathfrak{B})$ by the group of order 3 generated by $\phi$.

Now suppose that $\mathfrak{F}$ is not algebraically closed, and let $\Re$ be its algebraic closure. The unique extensions of $\phi$ and $\sigma$ to automorphisms of $R_{\Omega}$ will again be denoted by $\phi$ and $\sigma$, and in case $n=4$, again generate a group of six auto- 
morphisms of $\Omega_{\Omega}$, none of which, other than the identity, is an invariant automorphism of $\mathfrak{l}_{\Omega}$. These extensions are defined by (16) and (17), where the entries in the matrices now lie in $\Re$. If $\tau$ is an automorphism of $R$, then $\tau$ admits a unique extension to an automorphism of $\mathfrak{R}_{\Omega}$, which is thus of one of the forms $X^{\tau}=A^{-1} X A, X^{\tau}=A^{-1} X^{\phi} A, X^{\tau}=A^{-1} X^{\phi^{2}} A$, where $A \in O\left(\mathfrak{B}_{\Omega}\right)$ with respect to the extension to $\mathfrak{B}_{\Omega}$ of the form on $\mathfrak{B}$, and where the last two types occur if and only if $n=4$. If $x \in \mathfrak{R}$, then $X^{\phi} \in \mathfrak{R}$ and $X^{\phi^{2}} \in \mathfrak{R}$ if $n=4$; it follows by the result from [25] cited in previous cases that $\tau($ in $R)$ is of one of the forms $X^{\tau}=B^{-1} X B, X^{\tau}=B^{-1} X^{\phi} B, X^{\tau}=B^{-1} X^{\phi^{2}} B$, where $B \in \&(\mathfrak{B})$, the last two types occurring only when $n=4$. As before, we see that $B \in(B)$, the group of linear transformations $B$ of $\mathfrak{B}$ satisfying $(x B, y B)=(x, y) \beta$ for all $x, y \in \mathfrak{B}$, where $0 \neq \beta \in \mathfrak{F}$. Also as before, if $n \neq 4$ the group of automorphisms $\mathfrak{A}$ of $\mathfrak{Z}$ is isomorphic with $\mathbb{S} / \mathfrak{F}^{*} I$; if $n=4, \mathfrak{A}$ is the product of $\mathbb{S} / \mathfrak{F}^{*} I$ by the group of order 3 generated by $\phi$.

If $\mathfrak{S}_{0}$ is any standard Cartan subalgebra of $\mathfrak{R}$, and if $\mathfrak{S}$ is the standard Cartan subalgebra consisting of those transformations whose matrices relative to the basis $e_{1}, \cdots, e_{2 n}$ are diagonal, then it follows as in previous sections that there is an automorphism $\tau$ of $\mathscr{\ell}$ such that $\mathfrak{S}^{r}=\mathfrak{S}_{0}$. Since $\phi$ maps $\mathfrak{S}$ onto $\mathfrak{E}$, it follows that in every case there is a linear transformation $B$ of $\mathfrak{B}$ such that $(x B, y B)=(x, y) \beta$ for some fixed nonzero $\beta \in \mathfrak{F}$ and for all $x, y \in \mathfrak{B}$, and such that $B^{-1} \mathfrak{E} B=\mathfrak{S}_{0}$. Thus $\mathfrak{S}_{0}$ consists of the transformations of $\mathfrak{B}$ whose matrices relative to $e_{1} B, \cdots, e_{2 n} B$ are diagonal and which lie in $\&$, where $\left(e_{i} B, e_{j} B\right)=\left(e_{i}, e_{j}\right) \beta$ for all $i, j$. A basic set of root-vectors $E_{\alpha}$ relative to $\mathfrak{S}_{0}$ consists of the $E_{i j}-E_{j+n, i+n}, i \neq j ; E_{i, j+n}-E_{j, i+n}, i<j ; E_{i+n, j}-E_{j+n, i}$, $i<j ; 1 \leqq i, j \leqq n$, where the $E_{k m}$ are the matrix-unit transformations relative to the basis $e_{1} B, \cdots, e_{2 n} B$ for $\mathfrak{B}$. The group $G^{\prime}$ of Chevalley formed over $\mathfrak{F}$ from a complex Lie algebra of type $D_{n}(n \geqq 3)$ coincides with the group generated by all $\exp \left(\operatorname{ad} E_{\alpha}\right)$, where $E_{\alpha}$ runs through all root-vectors relative to a fixed Cartan subalgebra of $\mathfrak{R}$, which we may assume to be the $\mathfrak{S}_{0}$ above. Now $G^{\prime} \subseteq \Im$, and the generators for $G^{\prime}$ are the transformations

$$
X \rightarrow\left(\exp E_{\alpha}\right)^{-1} X\left(\exp E_{\alpha}\right),
$$

where $\exp E_{\alpha}$ runs through the transformations of $\mathfrak{B}$ whose matrices relative to $e_{1} B, \cdots, e_{2 n} B$ are of the forms

$$
\begin{array}{ll}
I+\lambda\left(E_{i j}-E_{j+n, i+n}\right) ; & I+\lambda\left(E_{i, j+n}-E_{j, i+n}\right) ; \\
I+\lambda\left(E_{i+n, j}-E_{j+n, i}\right) ; & 1 \leqq i, j \leqq n, i \neq j ; \lambda \in \mathfrak{F} .
\end{array}
$$

By computation of spinorial norms [1] one sees that all of these transformations are in the commutator subgroup $\Omega(\mathfrak{B})$ of $O(\mathfrak{B})$, and this property is independent of the choice of the standard Cartan subalgebra $\mathfrak{S}_{0}$. Thus $G^{\prime} \subseteq \Im \subseteq P \Omega(\mathfrak{B})$, the quotient of $\Omega(\mathfrak{B})$ by the scalar transformations in it.

As in $\$ 4$, it may be well to supplement [7] and [19] by sketching a proof that the transformations (18) generate $\Omega(\mathfrak{B})$. We do so for all $n \geqq 2$. For $n>2$, 
a procedure analogous to that indicated in $\$ 4$ enables us to reduce the problem of expressing an arbitrary element of $\Omega(\mathfrak{B})$ in terms of the generators (18) to the case where $\mathfrak{B}$ is $(2 n-2)$-dimensional of the same type; thus it is enough to treat the case $n=2$. Here if $T \in O(\mathfrak{B})$, post-multiplication by suitably chosen elements of the type (18) reduces $T$ to $U=\operatorname{diag}\left\{1, \alpha, 1, \alpha^{-1}\right\}$ or to $U=E_{11}+\alpha E_{24}+E_{33}+\alpha^{-1} E_{42}$. If $T \in O^{+}(\mathfrak{B})$, the latter is impossible, and if $T \in \Omega(\mathfrak{B})$, we have $U \in \Omega(\mathfrak{B}), \alpha=\delta^{2}$ from the spinorial norm mapping, and $U=\operatorname{diag}\left\{1, \delta^{2}, 1, \delta^{-2}\right\}$. Then

$$
\begin{aligned}
U= & \left(I+\left(E_{14}-E_{23}\right)\right)\left(I+\left(E_{12}-E_{43}\right)\right)\left(I+(\delta-1)\left(E_{41}-E_{32}\right)\right) \\
& \cdot\left(I+\delta^{-1}(1-\delta)\left(E_{21}-E_{34}\right)\right)\left(I+(-\delta)\left(E_{12}-E_{43}\right)\right)\left(I-\delta^{-1}\left(E_{13}-E_{23}\right)\right) \\
& \cdot\left(I+\delta^{-2}(\delta-1)\left(E_{21}-E_{34}\right)\right)\left(I+\delta(\delta-1)\left(E_{32}-E_{41}\right)\right)
\end{aligned}
$$

This completes the proof that $\Omega(\mathfrak{B})$ is generated by the transformations (18), and with it the result $G^{\prime}=\Im=P \Omega(\mathfrak{B})$. We have thus proved

Theroem 4. Let $\mathfrak{B}$ be a 2 -dimensional vector space $(n \geqq 3)$ over a field $\mathfrak{F}$ of characteristic different from $2,3,5,7$, equipped with a nondegenerate symmetric bilinear form $(x, y)$ of maximal index. Let $\mathbb{Q}$ be the Lie algebra of all linear transformations of $\mathfrak{B}$ which are skew with respect to $(x, y)$. Let $(B)$ be the group of all linear transformations $A$ of $\mathfrak{B}$ satisfying $(x A, y A)=(x, y) \alpha$ for all $x, y \in \mathfrak{B}$ and for some $\alpha \neq 0$ in $\mathfrak{F}$, and in case $n=4$, let $\phi$ be the automorphism of $\mathfrak{R}$ given by (16). Then if $n \neq 4$, every automorphism of $\mathbb{R}$ is of the form $X \rightarrow A^{-1} X A, A \in(b)$,

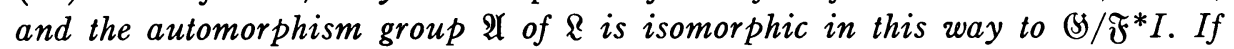
$n=4$, every automorphism is of one and only one of the forms $X \rightarrow A^{-1} X A$, $X \rightarrow A^{-1} X^{\phi} A, X \rightarrow A^{-1} X^{\phi^{2}} A$, so that $\mathfrak{\$} / \mathfrak{F}^{*} I$ is a subgroup of index 3 in $\mathfrak{A}$, and $\mathfrak{A}$ is the product of $\mathbb{S} / \mathfrak{F}^{*} I$ by the subgroup of order 3 generated by $\phi$. The group $\Im$ of invariant automorphisms is the group of all automorphisms $X \rightarrow U^{-1} X U$, where $U$ is in the commutator subgroup $\Omega(\mathfrak{B})$ of the orthogonal group $O(\mathfrak{B})$; thus $\Im$ is isomorphic with $P \Omega(\mathfrak{B})$ in all cases. The group $G^{\prime}$ of Chevalley formed over $\mathfrak{F}$ from a complex Lie algebra of type $D_{n}$ is the full group $\Im$, and is isomorphic to $P \Omega(\mathfrak{B})$.

6. Algebras of type $G$. Let $\mathfrak{F}$ be a field of characteristic $\neq 2, \mathfrak{B}=\mathfrak{B}_{3}(\mathfrak{F})$ the vector space of triples of elements of $\mathfrak{F}$ endowed with the usual scalar and vector products:

$$
\begin{aligned}
& \left(\alpha_{1}, \alpha_{2}, \alpha_{3}\right) \cdot\left(\beta_{1}, \beta_{2}, \beta_{3}\right)=\alpha_{1} \beta_{1}+\alpha_{2} \beta_{2}+\alpha_{3} \beta_{3}, \text { and } \\
& \left(\alpha_{1}, \alpha_{2}, \alpha_{3}\right) \wedge\left(\beta_{1}, \beta_{2}, \beta_{3}\right)=\left(\alpha_{2} \beta_{3}-\alpha_{3} \beta_{2}, \alpha_{3} \beta_{1}-\alpha_{1} \beta_{3}, \alpha_{1} \beta_{2}-\alpha_{2} \beta_{1}\right) .
\end{aligned}
$$

Then if $T \in \mathscr{E}(\mathfrak{B})$, one readily checks that

$$
(x \wedge y) \cdot z T+(y \wedge z) \cdot x T+(z \wedge x) \cdot y T=\operatorname{Sp}(T)(x \wedge y) \cdot z
$$

for all $x, y, z \in \mathfrak{B}$, where $S p(T)$ is the trace of $T$. If $T^{\prime}$ denotes the transpose of $T$ with respect to the scalar product $x \cdot y$, it follows from (19) and the identity $(x \wedge y) \cdot z=(y \wedge z) \cdot x$ that 


$$
(x \wedge y) T^{\prime} \cdot z+(x T \wedge y) \cdot z+(x \wedge y T) \cdot z=\operatorname{Sp}(T)(x \wedge y) \cdot z
$$

for all $x, y, z \in \mathfrak{B}$. Since the scalar product is nondegenerate, we have for all $x, y \in \mathfrak{B}$,

$$
(x \wedge y) T^{\prime}+(x T \wedge y)+(x \wedge y T)=\operatorname{Sp}(T)(x \wedge y) .
$$

The split Cayley algebra $\mathfrak{S}$ over $\mathfrak{F}$ may be defined as the 8-dimensional space of all matrices of the form

$$
\left(\begin{array}{ll}
\alpha & a \\
b & \beta
\end{array}\right)
$$

$\alpha, \beta \in \mathfrak{F} ; a, b \in \mathfrak{B}$; with the bilinear multiplication defined by

$$
\left(\begin{array}{ll}
\alpha & a \\
b & \beta
\end{array}\right)\left(\begin{array}{ll}
\gamma & c \\
d & \delta
\end{array}\right)=\left(\begin{array}{cc}
\alpha \gamma+a \cdot d & \alpha c+\delta a-b \wedge d \\
\gamma b+\beta d+a \wedge c & \beta \delta+b \cdot c
\end{array}\right) .
$$

The matrix

$$
I=\left(\begin{array}{ll}
1 & 0 \\
0 & 1
\end{array}\right)
$$

is evidently a unity element for this algebra. The algebra may be shown to be alternative and simple [27] - it is isomorphic to Zorn's algebra under

$$
\left(\begin{array}{ll}
\alpha & a \\
b & \beta
\end{array}\right) \rightarrow\left(\begin{array}{rr}
\alpha & -a \\
b & \beta
\end{array}\right)
$$

-and if for

$$
A=\left(\begin{array}{ll}
\alpha & a \\
b & \beta
\end{array}\right)
$$

we set

$$
\bar{A}=\left(\begin{array}{rr}
\beta & -a \\
-b & \alpha
\end{array}\right)
$$

then $A \rightarrow \bar{A}$ is an involution in $(\mathbb{S}$ over $\mathfrak{F}$ and $A \bar{A}=\bar{A} A=(\alpha \beta-a \cdot b) I$. We set $(A, A)=\alpha \beta-a \cdot b$. This form $(A, A)$ is then a quadratic form admitting composition, i.e., $(A B, A B)=(A, A)(B, B)$ for all $A, B \in \mathbb{C}$. The symmetric bilinear form resulting from polarization is $(A, B)$, defined by $A \bar{B}+B \bar{A}$ $=2(A, B) I=\bar{A} B+\bar{B} A$. One verifies easily that this form is nondegenerate. Now $(5$ has zero-divisors, e.g.,

$$
\left(\begin{array}{ll}
0 & a \\
0 & 0
\end{array}\right)^{2}=0
$$

for all $a \in \mathfrak{B}$; it is known that there is only one nonassociative algebra with unity over $\mathfrak{F}$ carrying a nonsingular quadratic form admitting composition 
and having zero-divisors [15]. Thus we have an abstract characterization of $\mathfrak{E}$; another may be given as a simple non-associative alternative algebra with zero-divisors [27]. The specific form used here will be advantageous in calculations with the derivation algebra, as well as in that it gives us a starting point independent of the other literature in this area.

Now let $\mathbb{R}$ be the Lie algebra of all derivations of $\mathfrak{S}$ over $\mathfrak{F} ; \mathfrak{R}$ is a restricted Lie algebra under $p$ th iterates if $\mathfrak{F}$ is of prime characteristic $p$. If $D \in \mathbb{R}$, it follows as usual that $I D=0$. Next let

$$
\left(\begin{array}{rr}
1 & 0 \\
0 & -1
\end{array}\right) D=\left(\begin{array}{ll}
\alpha & a \\
b & \beta
\end{array}\right)
$$

Then from

$$
\left(\begin{array}{rr}
1 & 0 \\
0 & -1
\end{array}\right)^{2}=I
$$

we have

$$
0=I D=\left(\begin{array}{rr}
1 & 0 \\
0 & -1
\end{array}\right)\left(\begin{array}{ll}
\alpha & a \\
b & \beta
\end{array}\right)+\left(\begin{array}{ll}
\alpha & a \\
b & \beta
\end{array}\right)\left(\begin{array}{rr}
1 & 0 \\
0 & -1
\end{array}\right)=2\left(\begin{array}{rr}
\alpha & 0 \\
0 & -\beta
\end{array}\right) .
$$

Thus

$$
\left(\begin{array}{rr}
1 & 0 \\
0 & -1
\end{array}\right) D=\left(\begin{array}{ll}
0 & a_{1} \\
a_{2} & 0
\end{array}\right), \quad a_{i} \in \mathfrak{B}
$$

If $0 \neq a \in \mathfrak{B}$, then

$$
\left(\begin{array}{ll}
0 & 0 \\
a & 0
\end{array}\right)^{2}=0
$$

so that if

$$
\left(\begin{array}{ll}
0 & 0 \\
a & 0
\end{array}\right) D=\left(\begin{array}{ll}
\beta & b \\
c & \gamma
\end{array}\right)
$$

we find

$$
0=\left(\begin{array}{ll}
0 & 0 \\
a & 0
\end{array}\right)\left(\begin{array}{ll}
\beta & b \\
c & \gamma
\end{array}\right)+\left(\begin{array}{ll}
\beta & b \\
c & \gamma
\end{array}\right)\left(\begin{array}{ll}
0 & 0 \\
a & 0
\end{array}\right)=\left(\begin{array}{cc}
a \cdot b & 0 \\
(\beta+\gamma) a & a \cdot b
\end{array}\right),
$$

so that

$$
\left(\begin{array}{ll}
0 & 0 \\
a & 0
\end{array}\right) D=\left(\begin{array}{rr}
\beta & b \\
c & -\beta
\end{array}\right)
$$

where $a \cdot b=0$. From 
1960] ON AUTOMORPHISMS OF LIE ALGEBRAS OF CLASSICAL TYPE. II 473

$$
\left(\begin{array}{ll}
0 & 0 \\
a & 0
\end{array}\right)\left(\begin{array}{rr}
1 & 0 \\
0 & -1
\end{array}\right)=\left(\begin{array}{ll}
0 & 0 \\
a & 0
\end{array}\right)
$$

we obtain

$$
\left(\begin{array}{cr}
\beta & b \\
c & -\beta
\end{array}\right)=\left(\begin{array}{ll}
0 & 0 \\
a & 0
\end{array}\right)\left(\begin{array}{ll}
0 & a_{1} \\
a_{2} & 0
\end{array}\right)+\left(\begin{array}{rr}
\beta & b \\
c & -\beta
\end{array}\right)\left(\begin{array}{rr}
1 & 0 \\
0 & -1
\end{array}\right)=\left(\begin{array}{cc}
\beta & -b-a \wedge a_{2} \\
c & \beta+a \cdot a_{1}
\end{array}\right),
$$

from which $\beta=-2^{-1} a \cdot a_{1}, b=-2^{-1} a \wedge a_{2}=2^{-1} a_{2} \wedge a$. Thus

$$
\left(\begin{array}{ll}
0 & 0 \\
a & 0
\end{array}\right) D=\frac{1}{2}\left(\begin{array}{cc}
-a \cdot a_{1} & a_{2} \wedge a \\
a T & a \cdot a_{1}
\end{array}\right), \quad T \in \mathbb{E}(\mathfrak{B}) .
$$

Similarly,

$$
\left(\begin{array}{ll}
0 & a \\
0 & 0
\end{array}\right) D=\frac{1}{2}\left(\begin{array}{cc}
-a \cdot a_{2} & a U \\
a_{1} \wedge a & a \cdot a_{2}
\end{array}\right), \quad U \in \mathbb{E}(\mathfrak{B}) .
$$

For $a, b \in \mathfrak{B}$,

$$
\left(\begin{array}{ll}
0 & a \\
0 & 0
\end{array}\right)\left(\begin{array}{ll}
0 & 0 \\
b & 0
\end{array}\right)=\left(\begin{array}{cc}
a \cdot b & 0 \\
0 & 0
\end{array}\right)=\frac{1}{2} a \cdot b\left[\left(\begin{array}{ll}
1 & 0 \\
0 & 1
\end{array}\right)+\left(\begin{array}{rr}
1 & 0 \\
0 & -1
\end{array}\right)\right],
$$

and applying $D$ yields

$$
\frac{1}{2}\left(\begin{array}{ll}
0 & a \\
0 & 0
\end{array}\right)\left(\begin{array}{cc}
-b \cdot a_{1} & a_{2} \wedge b \\
b T & b \cdot a_{1}
\end{array}\right)+\left(\begin{array}{cc}
-a \cdot a_{2} & a U \\
a_{1} \wedge a & a \cdot a_{2}
\end{array}\right)\left(\begin{array}{ll}
0 & 0 \\
b & 0
\end{array}\right)=\frac{1}{2} a \cdot b\left(\begin{array}{ll}
0 & a_{1} \\
a_{2} & 0
\end{array}\right) .
$$

From comparing entries in the $(1,1)$-position, we see that $a \cdot b T+a U \cdot b=0$, or $U=-T^{\prime}$.

Finally let $a, b \in \mathscr{B}$ with $a \wedge b \neq 0$. Then we have

$$
\left(\begin{array}{ll}
0 & 0 \\
b & 0
\end{array}\right)\left(\begin{array}{ll}
0 & 0 \\
a & 0
\end{array}\right)=\left(\begin{array}{cc}
0 & a \wedge b \\
0 & 0
\end{array}\right)
$$

so that applying $D$ gives

$$
\begin{aligned}
\frac{1}{2}\left(\begin{array}{rl}
-(a \wedge b) \cdot a_{2} & -(a \wedge b) T^{\prime} \\
\left.a_{1} \wedge(a \wedge b)\right) & (a \wedge b) \cdot a_{2}
\end{array}\right) \\
=\frac{1}{2}\left(\begin{array}{cc}
0 & 0 \\
b & 0
\end{array}\right)\left(\begin{array}{cc}
-a \cdot a_{1} & a_{2} \wedge a \\
a T & a \cdot a_{1}
\end{array}\right)+\left(\begin{array}{cc}
-b \cdot a_{1} & a_{2} \wedge b \\
b T & b \cdot a_{1}
\end{array}\right)\left(\begin{array}{ll}
0 & 0 \\
a & 0
\end{array}\right) \\
=\frac{1}{2}\left(\begin{array}{cc}
\left(a_{2} \wedge b\right) \cdot a & -b \wedge a T-b T \wedge a \\
-\left(a \cdot a_{1}\right) b+\left(b \cdot a_{1}\right) a & -b \cdot\left(a_{2} \wedge a\right)
\end{array}\right) .
\end{aligned}
$$

Comparing entries in the $(1,2)$-position gives $(a \wedge b) T^{\prime}+a T \wedge b+a \wedge b T=0$; but by (21), this is simply $\operatorname{Sp}(T)(a \wedge b)$, and $a \wedge b \neq 0$; thus $\operatorname{Sp}(T)=0$, and 
$T \in \mathbb{E}^{\prime}(\mathfrak{B})$, the space of linear transformations of $\mathfrak{B}$ of trace zero. From the above and the decomposition

$$
\left(\begin{array}{ll}
\alpha & a \\
b & \beta
\end{array}\right)=\frac{\alpha+\beta}{2}\left(\begin{array}{ll}
1 & 0 \\
0 & 1
\end{array}\right)+\frac{\alpha-\beta}{2}\left(\begin{array}{rr}
1 & 0 \\
0 & -1
\end{array}\right)+\left(\begin{array}{ll}
0 & a \\
0 & 0
\end{array}\right)+\left(\begin{array}{ll}
0 & 0 \\
b & 0
\end{array}\right)
$$

we have

$$
\left(\begin{array}{ll}
\alpha & a \\
b & \beta
\end{array}\right) D=\frac{1}{2}\left(\begin{array}{cc}
-a \cdot a_{2}-b \cdot a_{1} & (\alpha-\beta) a_{1}+\left(a_{2} \wedge b\right)-a T^{\prime} \\
(\alpha-\beta) a_{2}+\left(a_{1} \wedge a\right)+b T & a \cdot a_{2}+b \cdot a_{1}
\end{array}\right)
$$

where $a_{1}, a_{2}$ are fixed in $\mathfrak{B}$, and where $T \in \mathbb{E}^{\prime}(\mathfrak{B})$. By the computations above, $D$ uniquely determines $a_{1}, a_{2}$, and $T$, and the mapping $D \rightarrow\left(a_{1}, a_{2}, T\right)$ is linear from $\mathcal{R}$ into the direct sum $\mathfrak{B} \oplus \mathfrak{B} \oplus \mathscr{E}^{\prime}(\mathfrak{B})$. This direct sum is a 14-dimensional vector space over $\mathfrak{F}$, and the mapping is evidently one-one since $a_{1}, a_{2}, T$ determine $D$ uniquely by (22). We now show that the mapping is onto, hence that $\mathfrak{l}$ is 14 -dimensional. Therefore let $a_{1}, a_{2} \in \mathfrak{B}, T \in \mathbb{F}^{\prime}(\mathfrak{B})$, and define a linear transformation $D$ of $\mathbb{E}$ by (22). By use of the following identities, one readily verifies that $D$ is a derivation of $\mathfrak{E}$ :

$$
\begin{gathered}
a T \wedge b+a \wedge b T=-(a \wedge b) T^{\prime} ; \quad a \wedge b=-b \wedge a ; \quad a \cdot b=b \cdot a \\
(a \wedge b) \cdot c=(b \wedge c) \cdot a ; \quad(a \wedge b) \wedge c=(a \cdot c) b-(b \cdot c) a .
\end{gathered}
$$

From the effect of $D$ on

$$
\left(\begin{array}{rr}
1 & 0 \\
0 & -1
\end{array}\right) \text { and on }\left(\begin{array}{ll}
0 & 0 \\
a & 0
\end{array}\right)
$$

it is clear that the image of $D$ under the mapping of $\mathfrak{R}$ into $\mathfrak{B} \oplus \mathfrak{B} \oplus \mathbb{E}^{\prime}(\mathfrak{B})$ is $\left(a_{1}, a_{2}, T\right)$; thus our mapping is onto, and we use it to identify $\mathfrak{R}$ with $\mathfrak{B} \oplus \mathfrak{B}$ $\oplus \mathbb{F}^{\prime}(\mathfrak{B})$.

If $D=\left(a_{1}, a_{2}, T\right)$ and $E=\left(b_{1}, b_{2}, U\right)$ are in $\mathfrak{R}$, then $[D E] \in \mathfrak{R}$, so that $[D E]=\left(c_{1}, c_{2}, V\right)$ for some $c_{1}, c_{2} \in \mathfrak{B}, V \in \mathbb{E}^{\prime}(\mathfrak{B})$. From computing

$$
\left(\begin{array}{rr}
1 & 0 \\
0 & -1
\end{array}\right)[D E]=\frac{1}{2}\left(\begin{array}{cc}
0 & -a_{1} U^{\prime}+b_{1} T^{\prime}+2\left(b_{2} \wedge a_{2}\right) \\
a_{2} U-b_{2} T+2\left(b_{1} \wedge a_{1}\right) & 0
\end{array}\right) \text {, }
$$

we have

$$
\begin{aligned}
& c_{1}=b_{2} \wedge a_{2}-2^{-1} a_{1} U^{\prime}+2^{-1} b_{1} T^{\prime}, \\
& c_{2}=b_{1} \wedge a_{1}+2^{-1} a_{2} U-2^{-1} b_{2} T .
\end{aligned}
$$

If we let $x \in \mathfrak{B}$ and compute the $(2,1)$-entry in

$$
\left(\begin{array}{ll}
0 & 0 \\
x & 0
\end{array}\right)[D E]
$$

we find 


$$
\left.\left.x V=2^{-1}\left(b_{1} \wedge\left(a_{2} \wedge x\right)+2\right) x \cdot b_{1}\right) a_{2}-a_{1} \wedge\left(b_{2} \wedge x\right)-2\left(x \cdot a_{1}\right) b_{2}+x[T U]\right)
$$

Now let $e_{1}=(1,0,0), e_{2}=(0,1,0), e_{3}=(0,0,1)$ be the standard basis for $\mathfrak{B}$, and let $E_{i j}, 1 \leqq i, j \leqq 3$, be that element of $\&(\mathfrak{B})$ which sends $e_{i}$ into $e_{j}$ and $e_{k}$ into 0 if $k \neq i$. Then $\mathcal{R}$ has as basis the elements $\left(e_{i}, 0,0\right) ;\left(0, e_{i}, 0\right)$; $\left(0,0, E_{i j}\right), 1 \leqq i, j \leqq 3, i \neq j$; and $H_{i}=E_{i i}-E_{i+1, i+1}, i=1,2$. Then $E_{i j}^{\prime}=E_{j i}$. $i \neq j$, and $H_{i}^{\prime}=H_{i}$.

From (23) and (24), we see that if $f_{i}=2\left(0,0, H_{i}\right), i=1,2$, then $\left[f_{1} f_{2}\right]$ $=2\left(0,0,\left[H_{1}, H_{2}\right]\right)=0$. Let $\mathfrak{S}$ be the two-dimensional commutative subalgebra of $\mathfrak{Q}$ spanned by $f_{1}$ and $f_{2}$. For $j \neq k$, we have

$$
\left[E_{j k} H_{i}\right]=\left(\delta_{k i}-\delta_{k, i+1}-\delta_{j i}+\delta_{j, i+1}\right) E_{j k} .
$$

Set $e_{\alpha_{1}}=\left(0,0, E_{21}\right), e_{-\alpha_{1}}=\left(0,0, E_{12}\right)$. If we define a linear function $\alpha_{1}$ on $\mathfrak{S}$ by $\alpha_{1}\left(f_{1}\right)=2, \alpha_{1}\left(f_{2}\right)=-1$, we see from (23) and (24) that $\left[e_{\beta} f_{i}\right]=\beta\left(f_{i}\right) e_{\beta}$, $\beta= \pm \alpha_{1}$, or $\left[e_{\beta} h\right]=\beta(h) e_{\beta}$ for all $h \in \mathfrak{S}$. Next let $\alpha_{2} \in \mathfrak{S}^{*}$ with $\alpha_{2}\left(f_{1}\right)=-1$, $\alpha_{2}\left(f_{2}\right)=0$, and set $e_{\alpha_{2}}=\left(e_{1}, 0,0\right), e_{-\alpha_{2}}=\left(0, e_{1}, 0\right), e_{\alpha_{1}+\alpha_{2}}=\left(e_{2}, 0,0\right), e_{-\alpha_{1}-\alpha_{2}}$ $=\left(0, e_{2}, 0\right), e_{\alpha_{1}+2 \alpha_{2}}=\left(0, e_{3}, 0\right), e_{-\alpha_{1}-2 \alpha_{2}}=\left(e_{3}, 0,0\right), e_{\alpha_{1}+3 \alpha_{2}}=\left(0,0, E_{13}\right), e_{-\alpha_{1}-3 \alpha_{2}}$ $=\left(0,0, E_{31}\right), e_{2 \alpha_{1}+3 \alpha_{2}}=\left(0,0, E_{23}\right), e_{-2 \alpha_{1}-3 \alpha_{2}}=\left(0,0, E_{32}\right)$. Together with the $f_{i}$, these $e_{\beta}$ form a basis for $\mathfrak{R}$ and for each $\beta$ we have $\left[e_{\beta} h\right]=\beta(h) e_{\beta}$ for all $h \in \mathfrak{S}$.

If $x=\lambda_{1} f_{1}+\lambda_{2} f_{2}+\sum_{\beta} \lambda_{\beta} e_{\beta}$ is an arbitrary element of $\mathbb{R}$, where the summation runs over all twelve $\beta \in \mathfrak{S}^{*}$ as enumerated above, and if $[x h] \in \mathfrak{S}$ for all $h \in \mathfrak{S}$, we have $\sum_{\beta} \lambda_{\beta} \beta(h) e_{\beta}=0$ for all $h \in \mathfrak{S}$ and therefore $\lambda_{\beta} \beta(h)=0$ for all $\beta$ and for all $h \in \mathfrak{S}$. Since each $\beta \neq 0$, each $\lambda_{\beta}=0$, i.e., $x \in \mathfrak{W}$. Hence $\mathfrak{S}$ is its own normalizer in $\mathfrak{R}$, so is a commutative Cartan subalgebra of $\mathfrak{R}$. The Lie algebra $\mathfrak{R}$ is a direct sum of $\mathfrak{S}$ and of the one-dimensional rootspaces $\mathfrak{R}_{\beta}=\mathfrak{F} e_{\beta}$, and the twelve linear functions $\beta$ are readily seen to be distinct if the characteristic of $\mathfrak{F}$ is not 2 or 3 ; this is assumed in what follows. The roots clearly span $\mathfrak{S}^{*}$; in fact, $\alpha_{1}$ and $\alpha_{2}$ do so. Thus if $[x \mathbb{R}]=0$ for some $x \in \mathbb{R}$, we have $[x \mathfrak{G}]=0$, hence $x \in \mathfrak{S}$ by the above. We also have $\left[e_{\beta} x\right]=\beta(x) e_{\beta}=0$ for all $\beta$, therefore $x=0$ by the fact that the roots span $\mathfrak{S}^{*}$; thus we have proved that the center of $\mathfrak{R}$ is zero. We have seen above that $\left[\mathfrak{R}_{\beta} \mathfrak{S}\right]=\mathfrak{R}_{\beta}$ for all $\beta$; since $\mathfrak{S}$ is spanned by $f_{1}=\left[e_{-\alpha_{1}} e_{\alpha_{1}}\right]$ and $f_{2}=\left[e_{2 \alpha_{1}+3 \alpha_{2}} e_{-2 \alpha_{1}-3 \alpha_{2}}\right]$, we see that $[R \mathbb{R}]=\mathfrak{R}$.

All the axioms of [18] for a Lie algebra of classical type with Cartan subalgebra $\mathfrak{S}$ and roots $\beta$ have now been established, except for (iv) and (v). It is enough to show each $\left[\mathfrak{Q}_{-\beta} \mathfrak{R}_{\beta}\right] \neq 0$ to establish (iv), since each $\mathfrak{R}_{\beta}$ is onedimensional. For $\beta= \pm \alpha_{1}, \pm\left(\alpha_{1}+3 \alpha_{2}\right), \pm\left(2 \alpha_{1}+3 \alpha_{2}\right)$, this result follows from $\left[E_{12} E_{21}\right]=H_{1},\left[E_{13} E_{31}\right]=H_{1}+H_{2},\left[E_{23} E_{32}\right]=H_{2}$, respectively, and from the formula (24). For $\beta= \pm \alpha_{2}$, we have $\left[\left(e_{1}, 0,0\right),\left(0, e_{1}, 0\right)\right]=(0,0, V)$ by $(23)$, where by (24), $x V=-e_{1} \wedge\left(e_{1} \wedge x\right)-2\left(x \cdot e_{1}\right) e_{1}$ for all $x \in \mathfrak{B}$; in particular, $e_{1} V$ $=-2 e_{1} \neq 0$, so that $V \neq 0$ and $\left[\mathfrak{R}_{-\beta} \mathfrak{R}_{\beta}\right] \neq 0$. A similar argument may be applied to $\pm\left(\alpha_{1}+\alpha_{2}\right)$ and $\pm\left(\alpha_{1}+2 \alpha_{2}\right)$, and (iv) follows.

By observation of the list of roots, one sees that there are no two roots $\alpha$ and $\beta$, with 0 included as an admissible value for $\alpha$ only, such that all 
$\alpha+k \beta$ are roots ( 0 admitted) for all natural numbers $k$. It follows that axiom (v) of [18] is satisfied. One also checks readily that $\alpha_{1}-\alpha_{2}$ is not a root, that is, that $\alpha_{1}$ and $\alpha_{2}$ are a simple system of roots of type $G_{2}$ in the sense of [18]. By Theorem 8 of [18], $\&$ is a (normal) simple Lie algebra. The system $\alpha_{1}, \alpha_{2}$ is a fundamental system of roots, the unique minimal root being $-2 \alpha_{1}-3 \alpha_{2}$. It follows that $\mathbb{R}$ is the exceptional simple Lie algebra of type $G_{2}$.

From the formula (22), we see that $\mathbb{R}$ maps $\mathbb{C}$ into the subspace $\mathbb{C}^{\prime}$ of vector-matrices of trace zero. Let

$$
\begin{gathered}
v_{1}=\left(\begin{array}{rr}
1 & 0 \\
0 & -1
\end{array}\right), \\
v_{i+1}=\left(\begin{array}{ll}
0 & e_{i} \\
0 & 0
\end{array}\right), \quad v_{i+4}=\left(\begin{array}{ll}
0 & 0 \\
e_{i} & 0
\end{array}\right), \quad 1 \leqq i \leqq 3 .
\end{gathered}
$$

Then $v_{1}, \cdots, v_{7}$ are a basis for $\mathcal{E}^{\prime}$, and the matrices relative to this basis of the restrictions to $\mathbb{S}^{\prime}$ of our basis of $\mathfrak{R}$ are scalar multiples of the following:

$$
\begin{array}{ll}
\left(e_{1}, 0,0\right): 2 E_{12}+E_{37}-E_{46}-E_{51} ; & \left(0,0, E_{12}\right): E_{32}-E_{66} ; \\
\left(e_{2}, 0,0\right): 2 E_{13}-E_{27}+E_{45}-E_{61} ; & \left(0,0, E_{13}\right): E_{42}-E_{57} ; \\
\left(e_{3}, 0,0\right): 2 E_{14}+E_{26}-E_{35}-E_{71} ; & \left(0,0, E_{23}\right): E_{43}-E_{67} ; \\
\left(0, e_{1}, 0\right): 2 E_{15}-E_{21}+E_{64}-E_{73} ; & \left(0,0, E_{21}\right): E_{23}-E_{65} ; \\
\left(0, e_{2}, 0\right): 2 E_{16}-E_{31}-E_{54}+E_{72} ; & \left(0,0, E_{31}\right): E_{24}-E_{75} ; \\
\left(0, e_{3}, 0\right): 2 E_{17}-E_{41}+E_{53}-E_{62} ; \quad\left(0,0, E_{32}\right): E_{34}-E_{76} ; \\
\left(0,0, H_{1}\right): E_{22}-E_{33}-E_{55}+E_{66} ; \quad\left(0,0, H_{2}\right): E_{33}-E_{44}-E_{66}+E_{77} .
\end{array}
$$

Noting that these are linearly independent, we see that $\mathbb{R}$ may be identified with its restriction to $\mathbb{S}^{\prime}$. In this sense, one finds by computing products of the above that $\mathfrak{E}\left(\mathfrak{S}^{\prime}\right)=\mathfrak{R}+\mathfrak{R}^{2}+\mathfrak{R}^{3}$ (while $\left.\mathfrak{E}\left(\mathfrak{S}^{\prime}\right) \neq \mathfrak{F} I+\mathfrak{l}+\mathfrak{R}^{2}\right)$. For easier reference, we number this observation:

$$
\mathfrak{E}\left(\mathbb{S}^{\prime}\right)=\mathfrak{R}+\mathfrak{R}^{2}+\mathfrak{R}^{3}=\mathfrak{R}^{3} .
$$

We also have $\left(\operatorname{ad} E_{\alpha}\right)^{4}=0$ for all root-vectors $E_{\alpha}$ relative to standard Cartan subalgebras. It follows by Lemma 1 that $E_{\alpha}^{10}=0$ for all such $E_{\alpha}$. Thus all results of $\$ 1$ except for $(7)$ are valid when the characteristic of $\mathfrak{F}$ is different from $2,3,5,7,11,13,17$. Since $I D=0$ for all $D \in \mathcal{R}$, we have $E_{\alpha}^{10}=0$ on all of (5. Hence by (5) and (2), exp $E_{\alpha}$ is an automorphism of $\mathfrak{E}$, and the group $\Im$ of invariant automorphisms of $R$ is a subgroup of the group of automorphisms of $\mathfrak{l}$ of the form $X \rightarrow A^{-1} X A$, where $A$ is an automorphism of $\mathfrak{E}$; every such mapping is an automorphism of $\mathbb{R}$.

If $A$ is an automorphism of $\left(5\right.$, then $I A=(I A) I=(I A)\left(I A^{-1} A\right)=\left(I\left(I A^{-1}\right)\right) A$ $=\left(I A^{-1}\right) A=I$; thus $A$ is the identity on $\mathfrak{F} I$. We note also that if $x \in \mathbb{C}$, then $x^{2}-(x+\bar{x}) x+\bar{x} x=0$, and that $x+\bar{x}$ and $\bar{x} x$ are in $\mathfrak{F} I$. Hence, operating on 
this equation with $A$ and comparing the corresponding equation for $x A$ gives $\left({ }^{2}\right)$ $(x A)^{2}-(x+\bar{x}) x A+\bar{x} x=0=(x A)^{2}-\left(x A+(x A)^{-}\right) x A+(x A)^{-} x A$. In particular if $x \in \mathfrak{S}^{\prime}$, i.e., if $x+\bar{x}=0$, we obtain from canceling $(x A)^{2}$ that $\left(x A+(x A)^{-}\right) x A$ $=\left((x A)^{-}\right)(x A)-\bar{x} x \in \mathfrak{F} I$, with $x A+(x A)^{-} \in \mathfrak{F} I$. Then $x A \in \mathfrak{F} I$ unless $x A$ $+(x A)^{-}=0$, and since $A^{-1}$ maps $\mathfrak{F} I$ onto itself, we have $x \in \mathfrak{F} I$ unless $x A+(x A)^{-}=0 ;$ since $x+\bar{x}=0$ it follows from $x \in \mathfrak{F} I$ that $x=0$ and $x A+(x A)^{-}$ $=0$ in this case as well. Thus $x A+(x A)^{-}=0$ for all $x \in \mathbb{S}^{\prime}$, i.e., $A$ maps $\widetilde{C}^{\prime}$ in to $\mathbb{C}^{\prime}$. Since $x A+\bar{x} A=(x+\bar{x}) A=0$, we have $\bar{x} A=(x A)^{-}$for all $x \in \mathbb{C}^{\prime}$; this equation is evident for $x=I$, so by linearity $\bar{x} A=(x A)^{-}$for all $x \in \mathbb{E}$. Now let $A$ and $B$ be automorphisms of $\mathbb{C}$, and let $A^{-1} X A=B^{-1} X B$ for all $X \in \mathbb{R}$. Then $A$ and $B$ map $\mathbb{S}^{\prime}$ into $\mathbb{S}^{\prime}$, and $A B^{-1}$ is in the center of $\mathbb{E}\left(\mathbb{S}^{\prime}\right)$, by (26). Therefore $B=\lambda A$ on $\mathfrak{S}^{\prime}, \lambda \in \mathfrak{F}$. In particular, $\lambda v_{2} A=v_{2} B=\left(v_{1} v_{2}\right) B=\left(v_{1} B\right)\left(v_{2} B\right)$ $=\lambda^{2}\left(v_{1} A\right)\left(v_{2} A\right)=\lambda^{2}\left(v_{1} v_{2}\right) A=\lambda^{2} v_{2} A$, and since $\lambda \neq 0$ we have $\lambda=1, B=A$. Thus the homomorphism of the group $\mathfrak{A}(\mathfrak{E})$ of automorphisms of $\mathbb{E}$ into the group of automorphisms $\mathfrak{A}$ of $\mathfrak{R}$ sending $A \in \mathfrak{A}(\mathfrak{E})$ into the mapping $X \rightarrow A^{-1} X A$ is an isomorphism of $\mathfrak{A}(\mathfrak{E})$ into $\mathfrak{A}$.

In case the base field $\mathfrak{F}$ is algebraically closed, we know that $\mathfrak{F}=\mathfrak{A}$, hence that the inclusions $\mathfrak{Y} \subseteq \mathfrak{A}(\mathfrak{E}) \subseteq \mathfrak{A}$ are in fact equalities. Now suppose that $\mathfrak{F}$ is not algebraically closed, and let $\Omega$ be its algebraic closure. Then $\mathfrak{S}_{\Omega}$ is the algebra defined over $\Omega$ in exactly the same way as $\mathbb{E}$ is defined over $\mathfrak{F}$, and $\Omega_{\Omega}$ is a 14-dimensional subalgebra of the Lie algebra of derivations of $\mathfrak{S}_{\Omega}$, hence is the full derivation algebra of $\mathfrak{C}_{\mathfrak{R}}$. If $\sigma$ is an automorphism of $\mathfrak{R}$, denote also by $\sigma$ its unique extension to an automorphism of $\mathfrak{R}_{\Omega}$. Then there is an automorphism $A$ of $\mathfrak{夭}_{\Omega}$ such that $X^{\sigma}=A^{-1} X A$ for all $X \in \Omega_{\Omega}$, and so by [25] there is a nonsingular linear transformation $B$ of $\mathbb{E}$ such that $X^{\sigma}=B^{-1} X B$ for all $X \in \mathbb{R}$. Now we have $(I B)\left(B^{-1} X B\right)=I X B=O B=0$ for all $X \in \mathbb{R}$. Since the $B^{-1} X B$, restricted to $\mathbb{E}^{\prime}$, constitute the restriction of $L$ to $\mathbb{C}^{\prime}$, and so generate $\mathbb{E}\left(\mathbb{S}^{\prime}\right), I B$ cannot have any component in $\mathbb{S}^{\prime}$ in the direct decomposition $C=\mathfrak{F} I \oplus \mathbb{C}^{\prime}$. Thus $I B=\beta I, \beta \in \mathfrak{F}$. If $y \in \mathbb{S}^{\prime}$, then by the fact that the restriction of $\mathbb{R}$ to $\mathbb{S}^{\prime}$ generates $\mathbb{E}\left(\mathbb{S}^{\prime}\right)$ in the strong sense indicated by $(26)$, we can write $y=\sum x_{i} B^{-1} X_{i}, x_{i} \in \mathbb{C}, X_{i} \in \mathbb{R}$. Then $y B=\sum x_{i} B^{-1} X_{i} B=\sum x_{i} X_{i}^{\sigma}$ has trace zero, i.e., is in $\mathbb{S}^{\prime}$, since $\mathbb{R}$ maps $\mathbb{E}$ into $\mathbb{S}^{\prime}: \mathbb{S}^{\prime} B \subseteq \mathbb{S}^{\prime}$. Thus, restricting our attention to $\mathbb{C}_{\Omega}^{\prime}=\left(\mathbb{E}^{\prime}\right)_{\Omega}$, we have $B=\lambda A, \lambda \in \Omega$, by the fact that $\mathbb{R}_{\Omega}$ generates $\mathfrak{E}\left(\mathbb{G}_{\Re}^{\prime}\right)$. But we have $\left(v_{1} B\right)\left(v_{2} B\right)=\lambda^{2}\left(v_{1} A\right)\left(v_{2} A\right)=\lambda^{2}\left(v_{1} v_{2}\right) A=\lambda^{2} v_{2} A=\lambda v_{2} B \neq 0$. Since $\left(v_{1} B\right)\left(v_{2} B\right)$ and $v_{2} B$ are in $\mathfrak{S}, \lambda \in \mathfrak{F}$. Then $A=\lambda^{-1} B$ on $\mathfrak{S}^{\prime}$. If we define a nonsingular transformation $C$ of $\mathbb{E}$ by $I C=I, x C=\lambda^{-1} x B$ if $x \in \mathbb{S}^{\prime}$, then $C$ coincides with $A$ on $\mathfrak{E}$, hence is an automorphism of $\mathfrak{E}$, and $X^{\sigma}=C^{-1} X C$ for all $X \in \mathfrak{R}$. Thus we see that the full automorphism group $\mathfrak{A}$ of $\mathfrak{R}$ is again isomorphic to $\mathfrak{A}(\mathfrak{C})$.

If $\mathfrak{S}_{0}$ is any standard Cartan subalgebra of $\mathfrak{R}$, we see as before that $\mathfrak{S}_{0}$ is spanned by the two transformations whose matrices are $E_{22}-E_{33}-E_{55}+E_{66}$

(2) For convenience in printing, we write $(x A)^{-}$for the image of $x A$ under the involution $x \rightarrow \bar{x}$. 
and $E_{33}-E_{44}-E_{66}+E_{77}$ relative to a basis $v_{1} A, \cdots, v_{7} A$ for ( $^{\prime}$, where $A$ is an automorphism of $\mathbb{E}$. A basic set of root-vectors relative to $\mathfrak{S}_{0}$ consists of twelve derivations of $\mathbb{S}$ whose matrices relative to this new basis for $\mathbb{C}^{\prime}$ are the twelve corresponding to the root-vectors in (25). Chevalley's group $G^{\prime}$ formed from a complex Lie algebra of type $G_{2}$ may be identified with the subgroup of $\mathfrak{A}(\mathfrak{E})$ generated by the transformations of $\mathbb{S}$ whose matrices relative to one such basis for $\mathbb{E}$ are the exponentials of all scalar multiples of this set of matrices, namely:

(1') $I+\lambda\left(2 E_{12}+E_{37}-E_{46}-E_{51}\right)-\lambda^{2} E_{52}$;

(2') $I+\lambda\left(2 E_{13}-E_{27}+E_{45}-E_{61}\right)-\lambda^{2} E_{63}$;

(3') $I+\lambda\left(2 E_{14}+E_{26}-E_{35}-E_{71}\right)-\lambda^{2} E_{74}$;

(4') $I+\lambda\left(2 E_{15}-E_{21}+E_{64}-E_{73}\right)-\lambda^{2} E_{25}$;

$\left(5^{\prime}\right) I+\lambda\left(2 E_{16}-E_{31}-E_{54}+E_{72}\right)-\lambda^{2} E_{36}$;

(6) $I+\lambda\left(2 E_{17}-E_{41}+E_{63}-E_{62}\right)-\lambda^{2} E_{47}$; $\left(10^{\prime}\right) I+\lambda\left(E_{23}-E_{66}\right)$;

(11') $I+\lambda\left(E_{24}-E_{76}\right)$;

$\left(12^{\prime}\right) I+\lambda\left(E_{34}-E_{76}\right)$;

where $\lambda \in \mathfrak{F}$.

Let $v_{0}=I$, and let $u_{i}=v_{i} A$ above, $0 \leqq i \leqq 7$. Let $T$ be any automorphism of $\mathbb{E}$; we shall show how to express $T$ as a product of transformations of the types $\left(1^{\prime}\right)-\left(12^{\prime}\right)$ above, thereby proving that $G^{\prime}=\mathfrak{A}(\mathfrak{E})$, and consequently that $\mathfrak{Y}=\mathfrak{A}(\mathfrak{S})=\mathfrak{A}$ since $G^{\prime} \subseteq \mathfrak{Y} \subseteq \mathfrak{A}(\mathfrak{S})=\mathfrak{A}$. We proceed by multiplying $T$ on the right by various of the set $\left(1^{\prime}\right)-\left(12^{\prime}\right)$ of proposed generators for $\mathfrak{A}(\mathfrak{E})$, and shall use the same notation for the resulting transformation as for the original one. Once $T$ is so reduced to the identity automorphism we shall be done. Let $(\alpha)$ be the matrix of $T$ relative to the basis $u_{0}, \cdots, u_{7}$ for $\mathfrak{E}$; we already know that $u_{0} T=I T=I=u_{0}$ and that $T$ maps $\mathbb{C}^{\prime}$ into $\mathbb{E}^{\prime}$, so that $\alpha_{00}=1, \alpha_{0 j}=0=\alpha_{j 0}$ if $j>0$, and this property will hold for every automorphism. First we note that if $\alpha_{23} \neq 0$, we can follow $T$ by $I+\alpha_{23}^{-1}\left(1-\alpha_{22}\right)\left(E_{32}-E_{56}\right)$, with the effect that $\alpha_{22}=1$ for the resulting transformation. A similar procedure may be followed if any of $\alpha_{24}, \alpha_{27}, \alpha_{26} \neq 0$, by using types $\left(8^{\prime}\right),\left(5^{\prime}\right)$, $\left(6^{\prime}\right)$, respectively. If $\alpha_{22} \neq 0$ and if $\alpha_{23}=0$, then we can follow $T$ by type (10') with $\lambda=1$ to obtain $\alpha_{23} \neq 0$ and then proceed as above to obtain $\alpha_{22}=1$. If $\alpha_{27}=0$ but $\alpha_{25} \neq 0$ we can follow $T$ by type (8') with $\lambda=1$ to obtain $\alpha_{27} \neq 0$ and then may proceed as above. If all $\alpha_{2 j}=0$ for $j \neq 1$, then $\alpha_{21} \neq 0$ since $T$ is nonsingular, and we may follow by type $\left(2^{\prime}\right)$ with $\lambda=1$ to make $\alpha_{23} \neq 0$ and then make $\alpha_{22}=1$ as above. Thus we may assume $\alpha_{22}=1$. Now if we follow $T$ by types $\left(2^{\prime}\right)$ and $\left(3^{\prime}\right)$ in succession with $\lambda=\alpha_{27}$ and $\lambda=-\alpha_{26}$, respectively we retain the property $\alpha_{22}=1$ and obtain $\alpha_{26}=0=\alpha_{27}$ in the result. Now following by types $\left(10^{\prime}\right)$ and $\left(11^{\prime}\right)$ in succession with $\lambda=-\alpha_{23}$ and $\lambda=-\alpha_{24}$ respectively yields a result with $\alpha_{22}=1, \alpha_{26}=\alpha_{27}=\alpha_{23}=\alpha_{24}=0$. Next, following by type (4') with $\lambda=\alpha_{21}$ yields a result with $\alpha_{22}=1, \alpha_{2 j}=0$ if $j \neq 2,5$. Now $v_{2}^{2}=0=v_{5}^{2}$, while $v_{2} v_{5}+v_{5} v_{2}=I$; thus $u_{2}^{2}=0=\left(u_{2} T\right)^{2}=\left(u_{2}+\alpha_{25} u_{5}\right)^{2}=\alpha_{25} I$. Hence $\alpha_{25}=0$ and $u_{2} T=u_{2}$. 
Now $\left(u_{1} T\right) u_{2}=\left(u_{1} T\right)\left(u_{2} T\right)=\left(u_{1} u_{2}\right) T=u_{2} T=u_{2}$, since $v_{1} v_{2}=v_{2}$, so that in $u_{1} T=\sum \alpha_{1 i} u_{i}$, we have $\sum \alpha_{1 i} u_{i} u_{2}=u_{2}$. Now $\sum \alpha_{1 i} v_{i} v_{2}$ is readily seen to be $\alpha_{11} v_{2}-\alpha_{13} v_{7}+\alpha_{14} v_{6}+\alpha_{15} 2^{-1}\left(v_{0}-v_{1}\right)$, so that the corresponding equation holds with each $v_{i}$ replaced by $u_{i}$. Thus $u_{2}=\alpha_{11} u_{2}-\alpha_{13} u_{7}+\alpha_{14} u_{6}+\alpha_{15} 2^{-1}\left(u_{0}-u_{1}\right)$; by the linear independence of the elements of $C$ occurring here we have $\alpha_{11}=1$, $\alpha_{13}=\alpha_{14}=\alpha_{15}=0$. Now we follow $T$ in succession by types $\left(5^{\prime}\right)$ and $\left(6^{\prime}\right)$ with $\lambda=-2^{-1} \alpha_{16}$ and $\lambda=-2^{-1} \alpha_{17}$, respectively; the property $u_{2} T=u_{2}$ is preserved, as are $\alpha_{11}=1, \alpha_{13}=\alpha_{14}=\alpha_{15}=0$, and now we have $\alpha_{16}=0=\alpha_{17}$. If we now follow by type (1') with $\lambda=-2^{-1} \alpha_{12}$, all these properties are preserved, and we now have $\alpha_{12}=0$, i.e., $u_{1} T=u_{1}$. Thus we may assume $u_{1} T=u_{1}, u_{2} T=u_{2}$.

From $v_{1} v_{5}=-v_{5}$, we have $u_{1} u_{5}=-u_{5}$. If $u_{5} T=\sum \alpha_{5 j} u_{j}$, we have $u_{1}\left(u_{5} T\right)$ $=\alpha_{51} u_{0}+\alpha_{52} u_{2}+\alpha_{53} u_{3}+\alpha_{54} u_{4}-\alpha_{55} u_{5}-\alpha_{56} u_{6}-\alpha_{57} u_{7}$. Since $u_{1} T=u_{1}$, this is $\left(u_{1} T\right)\left(u_{5} T\right)=\left(u_{1} u_{5}\right) T=-u_{5} T=-\sum \alpha_{5 j} u_{j}$. Thus $\alpha_{51}=\alpha_{52}=\alpha_{53}=\alpha_{54}=0, u_{5} T$ $=\alpha_{55} u_{5}+\alpha_{56} u_{6}+\alpha_{57} u_{7}$. Next we have $u_{2} u_{5}=2^{-1}\left(u_{0}+u_{1}\right)$, so that $u_{2}\left(u_{5} T\right)$ $=\left(u_{2} T\right)\left(u_{5} T\right)=\left(u_{2} u_{5}\right) T=2^{-1}\left(u_{0}+u_{1}\right) T=2^{-1}\left(u_{0}+u_{1}\right)$, or $\alpha_{55} 2^{-1}\left(u_{0}+u_{1}\right)$ $=2^{-1}\left(u_{0}+u_{1}\right)$, since $u_{2} u_{6}=u_{2} u_{7}=0$. Therefore $\alpha_{55}=1, u_{5} T=u_{5}+\alpha_{56} u_{6}+\alpha_{57} u_{7}$. Now if we follow $T$ successively by types $\left(7^{\prime}\right)$ and $\left(8^{\prime}\right)$ with $\lambda=\alpha_{56}$ and $\lambda=\alpha_{57}$ respectively, we retain the properties $u_{1} T=u_{1}$ and $u_{2} T=u_{2}$, and have a new transformation with $u_{5} T=u_{5}$. Thus we may assume $T$ leaves these three elements fixed.

Similarly, from $u_{1} u_{3}=u_{3}$ and $u_{3} u_{5}=0$ and the fact that $u_{1}$ and $u_{5}$ are fixed, we find that $u_{3} T=\alpha_{33} u_{3}+\alpha_{34} u_{4}$. If $\alpha_{34} \neq 0$, we may follow by type $\left(9^{\prime}\right)$ with a suitable value of $\lambda$ to make $\alpha_{33}=1$ and then by type (12') with $\lambda=-\alpha_{34}$ to obtain $u_{3} T=u_{3}$. Since types $\left(9^{\prime}\right)$ and $\left(12^{\prime}\right)$ leave $u_{1}, u_{2}, u_{5}$ fixed, the resulting transformation will leave these fixed. If $\alpha_{34}=0$ then $\alpha_{33} \neq 0$ and we may follow by type (12') with $\lambda=1$ to make $\alpha_{34} \neq 0$ and then carry out the above process. Hence we may assume $u_{1}, u_{2}, u_{3}, u_{5}$ fixed under $T$.

By the fact that $u_{1} u_{4}=u_{4}$ and $u_{4} u_{5}=0$, we obtain as above $u_{4} T=\alpha_{43} u_{3}$ $+\alpha_{44} u_{4}$. Now $v_{3} v_{4}=v_{5}$ so that $\left(u_{3} u_{4}\right) T=u_{3}\left(u_{4} T\right)=u_{5} T=u_{5}$, or $u_{5}=\alpha_{44} u_{3} u_{4}$ $=\alpha_{44} u_{5}$, and $\alpha_{44}=1$. We follow by type $\left(9^{\prime}\right)$ with $\lambda=-\alpha_{43}$, and obtain $u_{4} T$ $=u_{4}$. Therefore we may assume $u_{1}, u_{2}, u_{3}, u_{4}, u_{5}$ fixed. Since $u_{2} u_{3}=u_{7}$ and $u_{4} u_{2}=u_{6}$, we see by the fact that $T$ is an automorphism that $T$ is the identity. Our reduction is therefore complete, and with it the proof that $G^{\prime}=\mathfrak{Y}=\mathfrak{A}$ (夭্) $=\mathfrak{A}$. We summarize in the following theorem:

Theorem 5. Let $\mathfrak{c}$ be the split Cayley algebra over a field $\mathfrak{F}$ of characteristic $\neq 2,3$. Let $\mathfrak{R}$ be the Lie algebra of derivations of $\mathfrak{C}$. Then $\mathfrak{R}$ is a simple Lie algebra of classical type $G_{2}$. If the characteristic of $\mathfrak{F}$ is not $5,7,11,13,17$, then the group $\Im$ of invariant automorphisms of $\&$ coincides with the full group $\mathfrak{A}$ of automorphisms of $\mathfrak{R}$, and the mapping sending $A \in \mathfrak{A}(\mathfrak{E})$ into the automorphism $X \rightarrow A^{-1} X A$ of $\mathfrak{R}$ is an isomorphism of the automorphism group $\mathfrak{A}(\mathfrak{S})$ of $\mathfrak{E}$ onto $\mathfrak{A}$. The group $G^{\prime}$ of Chevalley formed over $\mathfrak{F}$ from a complex Lie algebra of type $G_{2}$ coincides with $\Im$, hence is isomorphic to $\mathfrak{A}(\mathfrak{C})$.

7. Remarks. The exception of a number of low characteristics, necessi- 
tated by our use of the methods of $\S 1$, is in general unnecessary. In [14], Jacobson has used representation-theoretic methods (which are somewhat more cumbersome) to obtain the corresponding results on the full automorphism groups for all types $A-D$ except for $A_{n}$ when $p \mid(n+1)$ and for $D_{4}$. From these results and the fact that all $E_{\alpha}^{3}=0$ for these algebras, where $E_{\alpha}$ runs through the roots relative to the diagonal Cartan subalgebra, we see that the third powers are zero for all root-vectors relative to all standard Cartan subalgebras. Thus all our results carry over to characteristics $\neq 2,3$, with the exceptions of those on the $A_{n}$ and $D_{4}$ cited above. For algebras of type $G_{2}$, we can use unpublished work of C. W. Curtis on the irreducible restricted representations of restricted Lie algebras of classical type to show that an algebra of type $G_{2}$ has only one 7-dimensional irreducible restricted representation, and none of lower degree. It then follows as in $[13 ; 14 ; 24]$ that the full automorphism group of $G_{2}$ is as we have indicated above for all characteristics $\neq 2,3$, and also that $E_{\alpha}^{3}=0$ for all root-vectors $E_{\alpha}$ relative to standard Cartan subalgebras. Then again all our results on $G_{2}$ are valid for characteristics $\neq 2,3$. The number of cases to be considered in this calculation is rather formidable, and becomes nearly impossible for the other exceptional algebras.

In all cases so far investigated, the group $\Im$ of invariant automorphisms coincides with the group $G^{\prime}$, and is a known simple group $[1 ; 15]$. Moreover, $\Im$ is a normal subgroup of the full automorphism group $\mathfrak{A}$. It will be noted that in each case we have obtained an inclusion $\mathfrak{Y} \subseteq \mathfrak{B} \subseteq \mathscr{A}$, where $\mathfrak{B}$ is a group known to be simple. This could be used to show that $\Im=\mathscr{B}$ in these cases. However, we shall later encounter groups playing the role of $\mathfrak{B}$ where the simplicity does not yet seem to be established. The proof that $G^{\prime}=\mathfrak{Y}=\mathfrak{B}$ in these cases then establishes the simplicity of $\mathscr{B}$, since $G^{\prime}$ has been proved simple by Chevalley [3]. The same method of course yields the known results on simplicity of the groups $\mathfrak{B}$ occurring in this paper. The coincidence of $G^{\prime}$ and $\Im$ leads one to wonder whether it is possible to give a general proof that $G^{\prime}=\Im$, or at least that the group $G^{\prime}$ has all the properties attributed to $\Im$ in $(A)$. Another approach might be to attack $\Im$ from the point of view with which Chevalley has proved the simplicity of $G^{\prime}$.

Finally we indicate the nature of the quotient groups $\mathfrak{A} / \mathfrak{Y}$. For $A_{n-1}$ with $n>2$, we have $\mathfrak{A} / \mathfrak{\Im} \cong(\bar{\sigma}) \oplus\left(\mathfrak{F}^{*} /\left(\mathfrak{F}^{*}\right)^{n}\right)$, a direct sum of a cyclic group with generator $\bar{\sigma}$ of order 2 and the quotient of the multiplicative group $\mathfrak{F}^{*}$ of $\mathfrak{F}$ by the group of $n$th powers of elements of $\mathfrak{F}^{*}$. When $n=2$, we have $\mathfrak{A} / \mathfrak{Y}$ $\cong \mathfrak{F}^{*} /\left(\mathfrak{F}^{*}\right)^{2}$. If $\sigma$ denotes the automorphism $X \rightarrow-X^{\prime}$ and $I_{A}$ the automorphism $X \rightarrow A^{-1} X A$, then the isomorphism indicated is given by sending $\sigma^{i} I_{A}$ into $\bar{\sigma}^{i} \oplus\left(\operatorname{det}(A) \cdot \mathfrak{F}^{*^{n}}\right), j=1,2$, if $n>2 ; I_{A}$ into $\operatorname{det}(A) \cdot F^{* 2}$ if $n=2$.

For type $C_{n}$, the mapping $I_{A} \rightarrow \alpha \cdot F^{* 2}$, where $A \in(S)$ has $(x A, y A)=(x, y) \alpha$ for all $x, y \in \mathfrak{B}$, defines an isomorphism of $\mathfrak{A} / \mathfrak{S}$ onto $\mathfrak{F}^{*} / \mathfrak{F}^{* 2}$. For type $B_{n}$, every such $\alpha$ must be in $\mathfrak{F}^{* 2}$, hence there is a $U \in O(\mathfrak{B})$ such that $I_{A}=I_{U}$, and, 
replacing $U$ by $-U$ if necessary, we may assume $U \in O^{+}(\mathfrak{B})$. Now the spinorial norm mapping $\left[1\right.$, Chapter 5] maps $O^{+}(\mathfrak{B}) / \Omega(\mathfrak{B})$ isomorphically on to $\mathfrak{F}^{*} / \mathfrak{F}^{* 2}$. Since the only scalar in $O^{+}(\mathfrak{B})$ is the identity, we have $\mathfrak{A} \cong O^{+}(\mathfrak{B})$, and the spinorial norm mapping induces an isomorphism of $\mathfrak{A} / \mathfrak{Y}$ with $\mathfrak{F}^{*} / \mathfrak{F}^{* 2}$.

For type $D_{n}$, the situation is more complicated. First let $n \neq 4$. We have $\mathfrak{A}=I_{\mathfrak{G}}$, where the notation should be evident, and the mapping $I_{A} \rightarrow \alpha \mathfrak{F}^{* 2}$ as in type $C$ gives an isomorphism of $I_{\mathfrak{S}} / I_{O(\mathfrak{B})}$ onto $\mathfrak{F}^{*} / \mathfrak{F}^{* 2}$. Now $I_{O(\mathfrak{B})} / I_{O^{+}(\mathfrak{B})}$ is a group of order 2 generated by the coset of the automorphism $\sigma=I_{U}$ of $\S 5$, and $\Im=I_{\Omega(\mathfrak{B})}$. Now $-I \in O^{+}(\mathfrak{B})$, but $-I \in \Omega(\mathfrak{B})$ if and only if $(-1)^{n} \in \mathfrak{F}^{* 2}$, by Theorem 5.19 of $[1]$, and $(-1)^{n \mathfrak{F}^{* 2}}$ is the spinorial norm of $-I$. If $-I \in \Omega(\mathfrak{B})$, then the spinorial norm mapping induces an isomorphism of $I_{O^{+}(\mathfrak{B})} / I_{\Omega(\mathfrak{B})}$ onto $\mathfrak{F}^{*} / \mathfrak{F}^{* 2}$. If $-I \notin \Omega(\mathfrak{B})$, then $I_{\Omega(\mathfrak{B})} \cong \Omega(\mathfrak{B})$, and the homomorphism of $O^{+}(\mathfrak{B}) / \Omega(\mathfrak{B})$ onto $I_{o^{+}(\mathfrak{B})} / \Omega(\mathfrak{B})$ induces a homomorphism of $\mathfrak{F}^{*} / \mathfrak{F}^{* 2}$ onto a group isomorphic to the quotient group

$$
\left(\mathfrak{F}^{*} / \mathfrak{F}^{* 2}\right) /\left(\mathfrak{F}^{* 2},(-1)^{n} \mathfrak{F}^{* 2}\right)
$$

by a subgroup of two elements. If we agree to let the notation $\left(\mathfrak{F}^{* 2},(-1)^{n} \mathfrak{F}^{* 2}\right)$ mean the identity of $\mathfrak{F}^{*} / \mathfrak{F}^{* 2}$ when $(-1)^{n} \in \mathfrak{F}^{* 2}$, we have the normal series $\mathfrak{\Im} \subseteq I_{O+(\mathfrak{B})} \subseteq I_{O(\mathfrak{B})} \subseteq I_{\mathfrak{G}}=\mathfrak{A}$ with respective quotient groups

$$
\left(\mathfrak{F}^{*} / \mathfrak{F}^{* 2}\right) /\left(\mathfrak{F}^{* 2},(-1)^{n} \mathfrak{F}^{* 2}\right),
$$

a cyclic group $\mathfrak{Z}_{2}$ of order 2 , and $\mathfrak{F}^{*} / \mathfrak{F}^{* 2}$, as described above. The only change in this situation for $n=4$ is that $I_{\mathfrak{S}} \neq \mathfrak{A}$, and that $\mathfrak{A} / I_{\mathfrak{S}}$ is cyclic of order 3 , generated by the coset of $\phi$ of $\S 5$. Of course, $\mathfrak{A} / \Im$ is trivial in type $G_{2}$.

These results indicate that in general $\mathfrak{Y}$ is a larger subgroup of $\mathfrak{A}$ than we were able to prove in (A). It would be of interest to obtain directly the best possible result of this kind in the nonalgebraically-closed case, as was done in (A) for the algebraically closed case. One conjecture that seems to be indicated by the results is that conjugacy of standard Cartan subalgebras under $\mathfrak{Y}$ remains valid when $\mathfrak{F}$ is not algebraically closed. If this is correct, then a refinement of the methods used injproving Theorem 4 of $(\mathrm{A})$ would be a possible device for obtaining the final result.

\section{BiBLIOGRAPHY}

1. E. Artin, Geometric algebra, New York, Interscience, 1957.

2. E. Cartan, Les groupes projectifs qui ne laissent invariant aucune multiplicité plane, Bull. Soc. Math. France vol. 41 (1913) pp. 53-96. 66.

3. C. Chevalley, Sur certains groupes simples, Tôhoku Math. J. (2) vol. 7 (1955) pp. 14160-179.

4. C. W. Curtis, Modular Lie algebras. I, Trans. Amer. Math. Soc. vol. 82 (1956) pp.

5. - Modular Lie algebras. II, Trans. Amer. Math. Soc. vol. 86 (1957) pp. 91-108.

6. L. E. Dickson, Linear groups, Leipzig, Teubner, 1901.

7. - Theory of linear groups in an arbitrary field, Trans. Amer. Math. Soc. vol. 2 (1901) pp. 363-394. 
8. H. Freudenthal, Oktaven, Ausnahmegruppen und Oktavengeometrie, Mimeographed, Utrecht, 1951.

9. Harish-Chandra, On some applications of the universal enveloping algebra of a semisimple Lie algebra, Trans. Amer. Math. Soc. vol. 70 (1951) pp. 28-96.

10. N. Jacobson, Abstract derivation and Lie algebras, Trans. Amer. Math. Soc. vol. 42 (1937) pp. 206-224.

11. - Simple Lie algebras of type A, Ann. of Math. vol. 39 (1938) pp. 181-188.

12. - Simple Lie algebras over a field of characteristic zero, Duke Math. J. vol. 4 (1938) pp. 534-551.

13. - Cayley numbers and simple Lie algebras of type $G$, Duke Math. J. vol. 5 (1939) pp. $775-783$.

14. - Classes of restricted Lie algebras of characteristic p. I, Amer. J. Math. vol. 63 (1941) pp. 481-515.

15. - Composition algebras and their automorphisms, Rend. Circ. Mat. Palermo (2) vol. 7 (1958).

16. - Exceptional Lie algebras, Multilithed, New Haven, 1959.

17. W. H. Mills, Classical type Lie algebras of characteristic 5 and 7, J. Math. Mech. vol. 6 (1957) pp. 559-566.

18. W. H. Mills and G. B. Seligman, Lie algebras of classical type, J. Math. Mech. vol. 6 (1957) pp. 519-548.

19. R. Ree, On some simple groups defined by C. Chevalley, Trans. Amer. Math. Soc. vol. 84 (1957) pp. 392-400.

20. G. B. Seligman, On Lie algebras of prime characteristic, Memoirs, Amer. Math. Soc., no. $19,1956$.

21. - - Some remarks on classical Lie algebras, J. Math. Mech. vol. 6 (1957) pp. 549558.

22. - On automorphisms of Lie algebras of classical type, Trans. Amer. Math. Soc. vol. 92 (1959) pp. 430-448.

23. Séminaire "Sophus Lie." I, Mimeographed, Paris, 1954-1955.

24. M. L. Tomber, Lie algebras of type F, Proc. Amer. Math. Soc. vol. 4 (1953) pp. 759-768.

25. B. L. van der Waerden, Gruppen von linearen Transformationen, Ergebnisse der Math., Berlin, 1935; New York, Chelsea, 1948.

26. H. Weyl, Theorie der Darstellung kontinuierlicher halb-einfacher Gruppen durch lineare Transformationen. I, Math. Z. vol. 23 (1925) pp. 271-309.

27. M. Zorn, Theorie der alternativen Ringe, Abh. Math. Sem. Univ. Hamburg vol. 8 (1931) pp. 123-147.

Yale University, New Haven, Connecticut

UNIVERSITÄT MÜNSTER, MünSter, Germany 